\title{
Adhesive Friction Based on Accurate Elastic-Plastic Finite Element Analysis and $\mathrm{n}$-Point Asperity Concept
}

\author{
Ajay K. Waghmare and Prasanta Sahoo* \\ Deparment of Mechanical Engineering, Jadavpur University, Kolkata 700032, India \\ ${ }^{*}$ Corresponding author (Email: psjume@gmail.com)
}

Keywords: Adhesive friction; Elastic-plastic; Finite element method; n-Point asperity

\begin{abstract}
The present work considers analysis of adhesive friction of rough surfaces using n-point asperity concept for statistical definition of surface roughness features, and accurate finite element analysis of elastic-plastic deformation of single asperity contact. The paper describes theoretical study in which whole range of deformation of an n-point asperity viz. from fully elastic, through elastic-plastic, to fully plastic is considered and the intermediate transition regime is treated analytically as well as numerically. Well defined adhesion index and plasticity index are used to study the prospective contact situations arising out of variation in material properties and surface roughness features. Using practical values of material properties and surface roughness parameters, results are obtained for normally applied load, friction force, and coefficient of friction. It is observed that the surfaces undergoing predominantly plastic type of deformation and having moderate to higher adhesion have constant coefficient of friction.
\end{abstract}

\section{Introduction}

Friction as a phenomenon, is defined as a resistance to movement of one body over the other; and from the perspective of its quantification, friction represents the tangential resistive force on the contacting surfaces of the bodies which try to move relative to each other. Mindlin [1] in 1949 was the first who studied the Hertzian contact under combined influence of normal and tangential loading. He advocated the stick-slip theory of contact regime according to which there exist central stick region surrounded by annulus slip region. In mid-twentieth century, with the work of Bowden and Tabor, the direction of studying different contact related tribological phenomenon changed suddenly. Bowden and Tabor exemplified the role of surface roughness in contacting bodies. They introduced the concept of real area of contact and have shown that because of surface roughness the real area of contact is very small as compared to apparent area of contact. This understanding of contact area brought back the Coulomb's old idea of adhesion being a possible cause of friction. Later on in their classical theory of adhesive friction, Bowden and Tabor [2], described the adhesive friction as a tangential force required to shear off the adhesive bonds formed at the tip of contacting asperities due to local plastic deformation. Tabor [3] in his review paper presented a general critical picture of the understanding of frictional process. He emphasized that three basic elements are involved in the dry friction of solids viz. the true area of contact, the nature and strength of the interfacial bonds formed at the region of contact, and the way in which the material around the contact regions is sheared during sliding. Chang et al. [4] were the first to develop a static friction model for dry contact of rough surfaces by incorporating these three basic elements. They used Hamilton [5] explicit formulae to calculate the maximum tangential force that can be sustained before sliding inception. The Chang et al. approach of predicting tangential force differs from that of Mindlin with the fact that Chang et al. relates the start of full slip to material properties while Mindlin used local Coulomb friction law with a certain coefficient of friction. Again in Chang et al. model the first yielding of a single material point is treated as a criterion for sliding inception. Kogut and Etsion [6] showed that this assumption underestimates the permissible tangential force because the first failed point is still surrounded by considerable volume of elastic material which can support additional tangential load. Roy Chowdhury and Ghosh [7] used slip and yield as two separate limiting criterions to get total tangential force. They used Savkoor and Briggs [8] energy 
balance approach to get critical value of tangential force at which slip occurs while for plasticity condition they used von Mises criterion in conjunction with Hamilton stress field. In their model Roy Chowdhury and Ghosh assumed that with normally applied load some asperities get elastically deformed while others get plastically deformed and they ignored the intermediate elastic-plastic deformation of asperities. Chang, Etsion, and Bogy [9] (hence forth called as CEB approach) used conservation of asperity tip volume concept to account for area-displacement and load-displacement behaviours of the asperities undergoing transition type of deformation. But it is observed that the CEB analytical model suffers from a discontinuity in the contact load as well as in the first derivatives of contact load and contact area at elastic to elastic-plastic transition point. Literature shows some works (Evseev et al. [10]; Zhao et al. [11]; Kucharski et al. [12]) intended to remove the discrepancies found in CEB's model. But these works were either mere mathematical exercises without much relevance to physical contact phenomenon [10,11] or they lacked the accuracy [12]. Kogut and Etsion [13] (hence forth called as KE approach), based on their accurate FEA (Finite Element Analysis) results of single asperity contact, developed analytical expressions for transition zone behaviour of load and contact area.

Surface roughness plays significant role in the study of adhesion and concurrent adhesive friction (Roy Chowdhury and Ghosh [7]; Schaefer et al. [14]; Johnson [15]; Rabinovich et al. [16]). It is well known that the surface roughness is due to existence of protuberances of irregular size and shape and each such irregular entity found on the surface is called as an asperity. In conventional models the asperities are defined as peaks having some regular form at the tip. Also all such asperities on the surface are assumed to have identical tip radius with only variation in peak heights. This peak height concept of asperity signals that it is a three point asperity as minimum three points (height ordinates) are needed to define a peak. Though the models based on such asperity concept yield results in good agreement with experimental ones, fact remains that such definition of surface roughness features is far away from realistic. The n-point asperity concept introduced by Hariri, $\mathrm{Zu}$, and Ben Mrad in 2006 [17] uses such assumptions to define surface roughness features that it gives close to realistic picture of asperities. Models with n-point asperities assume that at particular level of separation between an interfering plane and a rough surface, there exist asperities of different sizes (i.e. different radii of curvatures and different heights). Also each asperity doesn't exist as a separate entity throughout the progression of contact but the earlier asperity gets merged into a new asperity with contact progression.

From above literature it is observed that scope exist to study adhesive frictional contact by combining the well established accurate contact analysis theories within the frame work of n-point asperity surface roughness features. In the present work an attempt is made to model adhesive friction at rough surface contact considering whole range of deformations and based on RG [7] slip and yield behaviour of asperities under combined normal and tangential loads; Savkoor and Briggs [8] energy balance approach to get friction force at slip; von-Mises theory in conjunction with Hamilton [5] stress field to get friction force at yield; CEB's analytical approach and KE's FEA approach to predict transition regime behaviour of deforming asperities; and Hariri et al. [17] npoint asperity frame work. To the best of authors knowledge, no such study is found in the existing literature. Based on this new n-point asperity model, the elastic-plastic normal contact problems for non-adhesive and adhesive contacts have been considered earlier by Hariri et al. [18], Sahoo et al. [19], and present authors [20-23].

\section{Fundamentals of n-Point Asperity Model}

The n-point asperity model developed by Hariri, $\mathrm{Zu}$, and Ben Mrad [17] is the foundation of present work. So in order to facilitate better understanding of present problem formulation, the npoint asperity concept is introduced in brief here. 


\section{n-Point Asperity Concept}

It is well known that the roughness of surface is due to existence of protuberances of irregular size and shape and each such irregular entity found on the surface is called as an asperity.

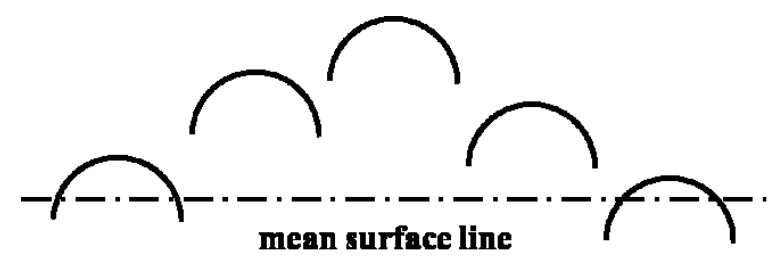

3-Point peak concept asperity (GW's asperity)

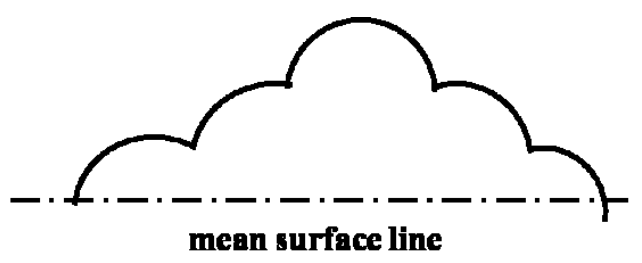

Peak on peak concept asperity (Archard's asperity) (a)

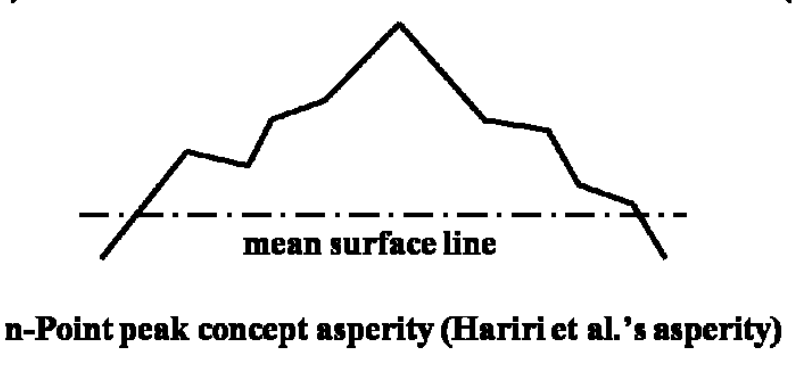

(b)

(c)

Figure 1. Illustration of different asperity concepts.

Fig. 1 illustrates the basic difference in definition of asperities by Greenwood and Williamson (GW) [24], Archard [25], and Hariri et al. [17]. In conventional model like GW, the asperities are defined as peaks having hemispherical tips. All such asperities on the surface are assumed to have identical tip radius but with variation in peak heights. This peak height concept of asperity signals that it is a three point asperity as minimum three points (height ordinates) are needed to define a peak. Most of the models found in the literature are mainly based on this three point peak model. In spite of the wide acceptance of this model, Greenwood and Wu [26] brought their original idea of three-point peak under question and called it inadequate because it gives false idea of both the radius of curvature of asperities and the number of asperities. They suggested considering asperities as protuberances on protuberances on protuberances which was originally proposed by Archard [25]. Hariri, $\mathrm{Zu}$, and Ben Mrad [17] used Archard's concept for definition of their asperity but with some modification. They used straight line segments to connect the consecutive height ordinates and obtained rough surface profile. The existence of an asperity on this profile is defined with respect to interference or separation between contacting surfaces. They named their asperity as npoint asperity where $n$ is the indicator of number of height ordinates of which the asperity is comprised. For statistical modeling of asperity topography, Hariri et al. used probability density function (pdf) to cover the variation in height of height ordinates while auto correlation function (ACF) is used to cover variation in spacing of height ordinates. Measured or practical values of standard deviation of height ordinates, correlation length, and correlation coefficient can be incorporated in the pdf and ACF to predict existence of n-point asperity topography on the surface. The concept of existence of an n-point asperity can be clearly understood from Fig. 2 and Fig. 3. 


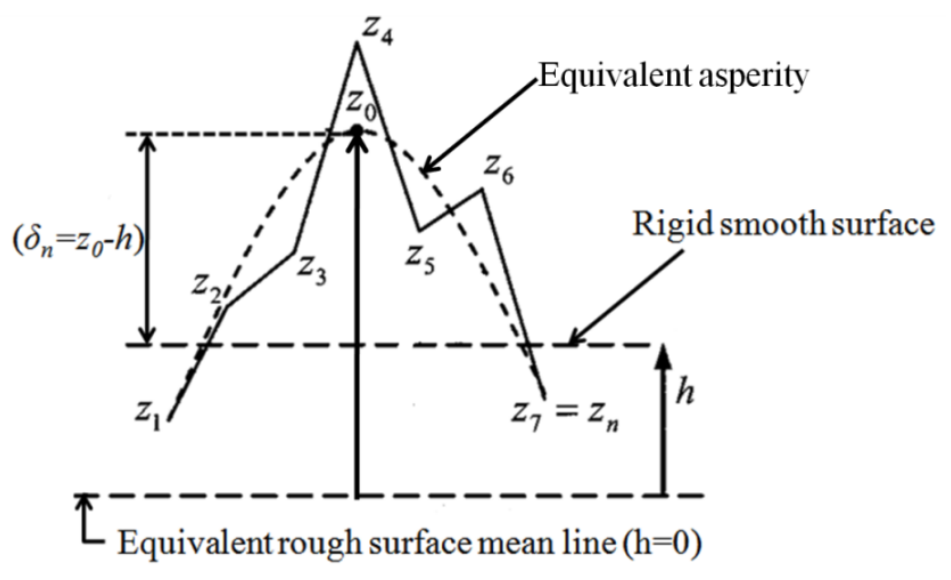

Figure 2. An n-point asperity and equivalent parabolic asperity.

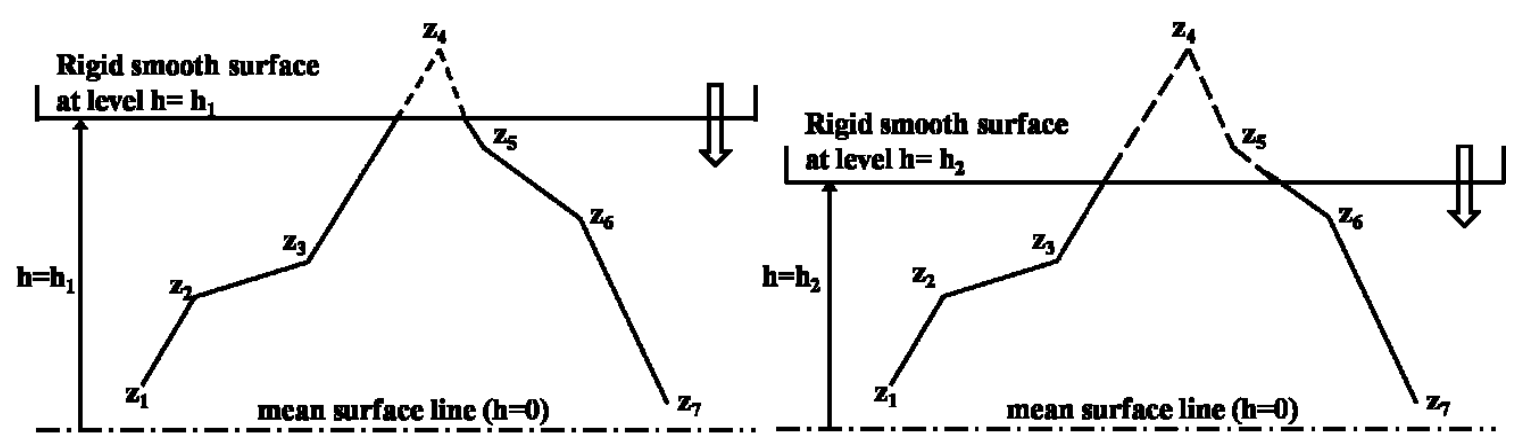

(a)

(b)

Figure 3. Progression of contact and emergence of new n-point asperity.

As per the definition (Hariri, $\mathrm{Zu}$, and Ben Mrad [17]), an n-point asperity is said to exist above a certain level from the mean line of surface profile when out of total ' $n$ ' consecutive peak ordinates, middle (n-2) ordinates are above the level and end two ordinates are below the level. As can be seen in the figure 2, the rigid smooth surface is at a level of ' $h$ ' from the mean line of rough surface profile and is interfering with the part of rough surface profile. Due such position of rigid smooth surface, there exist a 7-point asperity at a particular location on rough surface profile, which is comprised of total 7 number of consecutive height ordinates viz. $z_{1}, z_{2}, \ldots, z_{7}$. Out of which the middle ordinates $z_{2}, z_{3}, z_{4}$ and $z_{5}$ are above the level and the end ordinates $z_{1}$ and $z_{7}$ lie below the level. At a particular value of ' $h$ ', number of such asperities having different $n$ value will exist on the rough surface. But with the progression of rigid smooth surface, the size and number of n-point asperities on rough surface will change. Figure 3 shows progression of rigid smooth surface towards mean surface line of rough surface through an n-point asperity present on the rough surface. As can be seen in figures 3 (a) and (b), above separation level $h_{1}$ there exist 3-point asperity (asperity comprised of ordinates $z_{3}, z_{4}$ and $z_{5}$ ) while above separation level $h_{2}$ there exist 4-point asperity (asperity comprised of ordinates $z_{3}, z_{4}, z_{5}$, and $z_{6}$ ). Thus with the change in separation level, the asperity value ' $n$ ' as well as its size and shape changes. In short, the asperity curvature as well as height changes with the progression of contact, and with decrease in separation the previous asperity gets merged into a new asperity with higher number of n-points. Thus as compared to conventional Greenwood and Williamson model [24], the n-point asperity model represents the rough surfaces in more realistic form as it considers the variation in form of asperities in vertical direction (asperity height direction) as well as horizontal direction (asperity spacing direction). 


\section{Statistical Definition of an n-Point Asperity and Surface}

Statistically existence of an n-point asperity above a particular level ' $h$ ' from mean line of surface profile is defined as an event ' $S$ ' of height ordinates (points) such that $S=\left\{z_{1}<h, z_{2}, \ldots . ., z_{n-1}>h, z_{n}<h\right\}$. Where $z_{1}, z_{2}, \ldots, z_{n}$ are the consecutive height ordinates existing in such a fashion that middle $(n-2)$ ordinates are above the level and the two end ordinates are below the level. In statistical modeling, probabilistic approach is used to estimate the number of contacting asperities. Therefore, the probability of existence of an n-point asperity above predefined level ' $h$ ' will be same as the probability of existence of event $S$ and it can be given as

$$
N_{n}=\int_{z_{l}=-\infty}^{h} \int_{z_{2}=h}^{\infty} \ldots \int_{z_{i}=h}^{\infty} \ldots \int_{z_{n-l}=h}^{\infty} \int_{z_{n}=-\infty}^{h} f_{z_{1}, \ldots z_{n}}\left(z_{1}, \ldots z_{n}\right) d z_{n} d z_{n-1} . . d z_{i} \ldots d z_{2} d z_{1}
$$

where $f_{z_{1} \ldots \ldots . z_{n}}\left(z_{1}, \ldots . . z_{n}\right)$ is the joint probability density function (pdf) for event ' $S$ '. As the event ' $S$ ' consists of ' $n$ ' number of randomly varying height ordinates, each having its own distribution pattern, a joint pdf needs to be considered for the occurrence of event ' $S$ '. For the simplification of the problem, it is considered that each height ordinate follows Gaussian distribution. So the resultant joint pdf will also follow Gaussian distribution and as given by Hariri et al. [17], for exponential autocorrelation function with correlation coefficient $(\rho)$, it is given as

$$
f_{z_{1} \ldots z_{n}}\left(z_{1}, \ldots . . z_{n}\right)=\frac{e^{-z_{l}^{2} / 2}}{(2 \pi)^{n / 2}\left(1-\rho^{2}\right)^{(n-1) / 2}} \prod_{i=n-1}^{l} e^{-\left[\left(z_{i+1}-\rho z_{i}\right)^{2} / 2\left(l-\rho^{2}\right)\right]}
$$

If we consider total $M$ number of sample points on the surface then total number of n-point asperities in contact $\left(M_{n}\right)$ which are all comprised of same $n$ number of height ordinates will be obtained as

$$
M_{n}=M \times N_{n}
$$

If these $M$ sample points are obtained from a square grid formed by $(m \times m)$ perpendicular lines on a plane having separation between two consecutive lines as $\Delta x$, then $M=m^{2}$ and total nominal contact area $\left(A_{0}\right)$ of this grid will be given as

$$
A_{0}=\Delta x^{2} M
$$

Sampling length $(\Delta x)$ is function of correlation coefficient $(\rho)$ and correlation length $(\beta)$. For a rough surface on which sampling points are scattered by following exponential type of auto correlation pattern, the sampling length will be given as $\Delta x=(-\ln \rho) \times \beta$.

The statistical definition of an n-point asperity as given above depicts picture of discrete points which are the height ordinates. By connecting these height ordinates with lines, we get a polyline profile of rough surface. When a rigid smooth flat contacts with this polyline profile of rough surface, existence of n-point asperities of different dimensions get defined on the rough surface. The topography of an asperity gets more and more complicated with increase in value of ' $n$ ' and with progression of contact. As, such complex asperities lacks single curvature, formulation of contact analysis problems becomes more complicated. So it necessitates assigning equivalent single height and curvature to each n-point asperity. For this purpose, Hariri, Zu, and Ben Mrad [17] replaced the poly line form of an n-point asperity with an equivalent parabolic form which is shown as dotted line curve in the figure 2. The equivalent parabolic curve passes through the first and the last points and also through a middle imaginary point whose ordinate is given by $z_{0}$ (called equivalent asperity height). The criterion for fitting the parabolic curve is that the area under equivalent parabolic n-point asperity should be equal to the area under poly line form n-point 
asperity. The height $\left(z_{0}\right)$ and curvature $\left(C_{n}\right)$ of this equivalent parabolic n-point asperity according to Hariri, $\mathrm{Zu}$, and Ben Mrad [17] model are as given below.

$$
\begin{aligned}
z_{0} & =\frac{3}{2(n-1)} \sum_{i=2}^{n-1} z_{i}+\frac{4-n}{4(n-1)}\left(z_{1}+z_{n}\right), \text { for } n>3 \\
C_{n} & =\frac{2 z_{0}-z_{1}-z_{n}}{[(n-1) \Delta x / 2]^{2}}
\end{aligned}
$$

It should be noted that $z_{0}$ is simply equal to $z_{2}$ when $n=3$, and in such case above equation (5) need not be used.

\section{Modeling Adhesive Frictional Contact: CEB Approach}

In an earlier study [21], authors analysed the adhesive friction contact in the frame work of $n$ point asperities by following RG model where the total applied load is taken as mere contribution from purely elastic and purely plastic regimes of deformation. In the present section of this study an attempt is being made to take into account the contribution from elasto-plastically deforming transition zone asperities also. CEB, in their study of plain elastic-plastic contact makes use of conservation of asperity tip volume concept to account for elasto-plastic zone of deformation and the same is being used in this section of present study for adhesive friction contact. In short, with respect to existing $\mathrm{RG}$ model of adhesive frictional contact, the novelty of present section work is twofold, viz. the use of CEB approach of combining elastic and plastic parts of total applied load and modeling the contact in n-point asperity frame work.

\section{Applied Load Formulation for Single n-Point Asperity}

At any instance of contact, a rough surface will carry asperities showing different sort of deformations, viz. purely elastic, purely plastic, and elasto-plastic. In present section to formulate normally applied load, Johnson et al. [27] (hence forth called as JKR) theory is used for purely elastically deforming asperities, Roy Chowdhury and Pollock [28] theory is used for purely plastically deforming asperities, while CEB theory is used to account the effect of elasto-plastically deforming asperities. For a single n-point asperity pressed by a rigid flat, the applied load equations are

$$
\begin{aligned}
& \Delta P_{(e)_{n}}=\frac{K a_{(e)_{n}}^{3}}{R_{n}}-\left(6 \pi \gamma K a_{(e)_{n}}^{3}\right)^{\frac{1}{2}} \\
& \Delta P_{(p)_{n}}=\pi a_{(p)_{n}}^{2} H-2 \pi R_{n} \gamma
\end{aligned}
$$

In above equation (7), $\Delta P_{(e)_{n}}$ is the load supported by single n-point asperity having specific 'n' value in purely elastic domain, $K=\frac{4}{3} E, E$ is the composite elastic modulus given by $E=\left[\left(1-v_{1}^{2}\right) / E_{1}+\left(1-v_{2}^{2}\right) / E_{2}\right]^{-1}, E_{1}, E_{2}, v_{1}$ and $v_{2}$ being the elastic moduli and Poisson's ratios of the contacting surfaces respectively, $a_{(e)_{n}}$ is contact radius of an n-point asperity in elastic mode of deformation, $R_{n}$ is the equivalent radius of n-point asperity and it is the reciprocal of curvature as given in equation (6), i.e. $R_{n}=\frac{[(n-1) \Delta x / 2]^{2}}{c_{n} \sigma}$ with $c_{n}=\frac{2 z_{0}-z_{1}-z_{n}}{\sigma}$ as curvature coefficient, Following Hertz contact law, the contact radius of an n-point asperity in elastic mode of deformation is given by $a_{(e)_{n}}=\left(R_{n} \delta_{n}\right)^{l / 2}$ where interference of an n-point asperity is $\delta_{n}=z_{0}-h$. Here $z_{0}$ is the equivalent height of $n$-point asperity, $h$ is the mean separation. It has already been 
mentioned in the previous section that in n-point asperity model, the complex n-point asperity profile is converted into equivalent parabolic asperity profile. The quantitative parameters (equivalent height $z_{0}$ and radius $R_{n}$ ) of this best fit profile which is shown by dotted line in Fig. 2, are obtained by equating the areas under the profiles.

In above equation (8), $\Delta P_{(p)_{n}}$ can be used to calculate the load supported by single n-point asperity having specific ' $n$ ' value in either purely plastic or elasto-plastic domain depending upon the value of interference, $\delta_{n}$, of an asperity, $\mathrm{H}$, being the hardness of asperity material. Here contact radius $\left(a_{(p)_{n}}\right)$ of an n-point asperity in plastic mode of deformation is obtained by following the asperity tip volume conservation theory of Chang et al. [9], as

$$
a_{(p)_{n}}=\left(2 R_{n} \delta_{n}-R_{n} \delta_{(y)_{n}}\right)^{1 / 2}
$$

In above equation $\delta_{(y)_{n}}$ is the critical interference of an asperity tip at yielding inception. In the present study it is obtained by following Tabor [29] consideration, that at yield inception, maximum contact pressure on the body exceeds or at least becomes equal to 0.6 times its hardness. This plasticity condition, for an n-point asperity can be written as

$$
\frac{3}{2}\left(\frac{\Delta P_{(e)_{n}}}{\pi a_{(e)_{n}}^{2}}\right) \geq 0.6 H
$$

Above equation on expansion leads to

$$
\delta_{(a)_{n}}^{3 / 4}-0.94\left(\frac{H}{E}\right) R_{n}^{1 / 2} \delta_{(a)_{n}}^{1 / 4}-3.76\left(\frac{\gamma}{E}\right)^{1 / 2} R_{n}^{1 / 4} \geq 0
$$

In above equations $\delta_{n}$ and $\delta_{(a)_{n}}$ are real and apparent interferences of an n-point asperity. The apparent values of loading and deformation get introduced in to the analysis because of considerations of equivalence in behaviour of asperity under adhesive and non adhesive (purely Hertzian) conditions of contact [27].

Substituting the expression for $R_{n}$ which is in terms of $\beta, \rho, n, \sigma$ and $c_{n}$ in equation (11) and normalizing it with $\sigma$ (standard deviation of height ordinates or RMS height), we get following form of above equation.

$$
\delta_{(a)_{n}}^{* 3 / 4}-0.47(n-1)(-\ln \rho) \psi \frac{\delta_{(a)_{n}}^{* 1 / 4}}{c_{(a)_{n}}^{1 / 2}}-2.66\{(n-1)(-\ln \rho)\}^{1 / 2} \phi^{1 / 2} \frac{1}{c_{(a)_{n}}^{3 / 4}} \geq 0
$$

The parameter $c_{(a)_{n}}$ in above equation is the apparent value of curvature coefficient. As can be seen in this equation, the two new parameters viz. $\psi$ (plasticity index) and $\phi$ (adhesion index) get introduced in normalization of equation (11). The plasticity index $\psi=\frac{H \beta}{E \sigma}$ is same as defined by Hariri et al. [18], while the adhesion index $\phi=\frac{\gamma \beta}{E \sigma^{2}}$ is same as defined in Sahoo et al. [30].

Solution of equation (12) for lower limit of inequality gives the critical values (marking transition from elastic to plastic flow) of interference and curvature. But this single inequality is insufficient for solution, therefore two more relationships are considered. Following Johnson [31], the relationship between real and apparent values of interference for an n-point asperity can be given as

$$
\delta_{n}^{*}=\delta_{(a)_{n}}^{*}-1.77 \phi^{1 / 2}\{(n-1)(-\ln \rho)\}^{1 / 2} \frac{\delta_{(a)_{n}}^{* 1 / 4}}{c_{(a)_{n}}^{1 / 4}}
$$


As per Hariri et al. [18] and Sahoo et al. [30], at critical point of yielding inception, the curvature coefficient and interference are related as

$$
c_{(y)_{n}}=2 \delta_{(y)_{n}}^{*}
$$

Assuming the same relationship between their critical apparent values,

$$
c_{(y a)_{n}}=2 \delta_{(y a)_{n}}^{*}
$$

Now rewriting the equation (12) for critical point of yielding (i.e. by substituting $\delta_{(a)_{n}}^{*}=\delta_{(y a)_{n}}^{*}$, $\left.c_{(a)_{n}}=c_{(y a)_{n}}\right)$, and equating it to zero, we get

$$
\delta_{(y a)_{n}}^{* 3 / 4}-0.47(n-1)(-\ln \rho) \psi \frac{\delta_{(y a)_{n}}^{* 1 / 4}}{c_{(y a)_{n}}^{1 / 2}}-2.66\{(n-1)(-\ln \rho)\}^{1 / 2} \phi^{1 / 2} \frac{1}{c_{(y a)_{n}}^{3 / 4}}=0
$$

Solution of equations (13)-(16) gives critical values of real and apparent values of interference and curvature coefficient. In order to obtain a generalized solution, the load in equations (7) and (8) is first expanded to show the detailed geometric and material properties and then normalized with the load unit term $A_{0} H ; A_{0}$ being the total nominal contact area of rough surface and for a sample size of $M$ points it is as given by equation (4). In this normalization process, non dimensional indices viz. adhesion index $(\phi)$ and plasticity index $(\psi)$ get introduced in the load equations. For a single n-point asperity, expressions for non-dimensional load in elastic and plastic deformations of asperity are given by

$$
\begin{aligned}
& \Delta P_{(e)_{n}}^{*}=\frac{(n-1)}{(-\ln \rho)}\left\{\frac{0.67 \delta_{n}^{* 3 / 2}}{\psi c_{n}^{1 / 2}}-\frac{1.77(n-1)^{1 / 2}(-\ln \rho)^{1 / 2} \phi^{1 / 2} \delta_{n}^{* 3 / 4}}{\psi c_{n}^{3 / 4}}\right\} \\
& \Delta P_{(p)_{n}}^{*}=\frac{\pi(n-1)^{2}}{4}\left(\frac{\left(2 \delta_{n}^{*}-\delta_{(y)_{n}}^{*}\right)}{c_{n}}-\frac{2 \phi}{c_{n} \psi}\right)
\end{aligned}
$$

\section{Frictional Load Formulation for Single n-Point Asperity}

The classical theory of adhesive friction does not consider the effect of surface forces as for large-scale asperities adhesion is insignificant. On the other hand, for small scale asperities deformation and friction at very smooth and clean surfaces are strongly affected by surface forces. The frictional force for such asperities on the surface is the force offering resistance to tangential movement and plastically deformed asperities offer negligible resistance (Roy Chowdhury and Ghosh, [7]). The initially elastically deformed asperities contribute to friction force until yielding occurs under the combined action of normal load and tangential force. The other group of asperities which remain elastic even under the combined effect of normal and shear stresses will undergo slip. Thus the friction resistance for deformation up to plastic limit is composed of two parts: a) Friction resistance before tangential slip $\left(\Delta T_{\left(\text {slip }_{n}\right.}\right)$ and b) Frictional resistance before yielding $\left(\Delta T_{\left(\text {yield }_{n}\right.}\right)$. The frictional resistance for the asperities where slip occurs before yielding can be obtained using Savkoor and Briggs [8] energy balance approach, and the same when formulated for an n-point asperity by incorporating adhesion and plasticity indices and normalized with respect to load term $A_{0} H$ yields frictional resistance offered by an n-point asperity before slip in non dimensional form. It is given by

$$
\Delta T_{(s l i p)_{n}}^{*}=2.5\left(\frac{1-v}{2-v}\right)^{\frac{1}{2}}\left(\frac{\phi}{\psi}\right)\left\{\frac{(n-1)^{3}}{(-\ln \rho) \phi}\left(\frac{\delta_{n}^{*}}{c_{n}}\right)^{\frac{3}{2}}-1.77 \frac{(n-1)^{4}}{c_{n}^{2}}\right\}^{\frac{1}{2}}
$$


Beyond slip point there exist some asperities which are still elastic under combined effect of tangential and normal stresses and these asperities also contribute to frictional resistance until yielding takes place. So it becomes necessary to find out plasticity condition at the area of contact on an n-point asperity. It is obtained by using von-Mises criterion in conjunction with Hamilton [5] stress field. In his work Hamilton gives stress field for an elastic contact between two bodies with non-conforming surfaces, i.e. a sort of Hertzian contact, which in present study is treated as contact between an equivalent $n$-point asperity and a rigid flat. For a contact situation with contact area along $\mathrm{x}-\mathrm{y}$ plane, normal load $\Delta P_{(e)_{n}}$ along ' $\mathrm{z}$ ' direction and tangential load $\Delta T_{n}$ along ' $\mathrm{x}$ ' direction, Hamilton has shown that for a normally loaded contact the maximum tensile stress occurs at the circumference of the contact circle and in the presence of a tangential force in addition to the normal load the tensile stress increases at the back edge. Maximum shear stress, on the other hand, occurs below the surface for a normally loaded contact. An additional tangential force causes yielding to occur at lower loads and a new region of likely failure develops at the back edge of the contact circle. Beyond $\Delta T_{n} / \Delta P_{\left(e_{n}\right.} \approx 0.3$ this region dominates the stress field and below this value surface yielding occurs at even lower stress levels. It was therefore concluded that, on both counts, maximum tensile stress and maximum yield criteria, the region near the back edge of the contact is most likely point of failure. The stress field at this point for an elastically loaded n-point asperity is as given below.

$$
\left.\begin{array}{rl}
\sigma_{x} & =\frac{1-2 v}{2 \pi a_{(e)_{n}}^{2}} \Delta P_{(e)_{n}}+\frac{3 \Delta T_{n}}{4 a_{(e)_{n}}^{2}}\left(\frac{v}{4}+1\right) \\
\sigma_{y} & =\frac{9 v \Delta T_{n}}{16 a_{(e)_{n}}^{2}}-\frac{1-2 v}{2 \pi a_{(e)_{n}}^{2}} \Delta P_{(e)_{n}} \\
\sigma_{z} & =\tau_{x y}=\tau_{x z}=\tau_{z y}=0
\end{array}\right\}
$$

where the normally applied load $\Delta P_{(e)_{n}}$ and contact radius $a_{()_{n}}$ are the same as given by equation (7) in previous section. Using the above values of stresses in von-Mises plasticity criteria, the plasticity condition in non dimensional form for an n-point asperity works out as

$$
C_{1} \frac{\delta_{n}^{* 3}}{c_{n}}+C_{2} \frac{\delta_{n}^{* 9 / 4}}{c_{n}^{5 / 4}}+C_{3} \frac{\delta_{n}^{* 2}}{c_{n}^{2}}+\left(C_{4}+C_{5} \Delta T_{n}^{*} c_{n}\right) \frac{\delta_{n}^{* 3 / 2}}{c_{n}^{3 / 2}}+C_{6} \Delta T_{n}^{*} \frac{\delta_{n}^{* 3 / 4}}{c_{n}^{3 / 4}}+C_{7} \Delta T_{n}^{* 2}=0
$$

The constants in above equation $C_{1}, C_{2}$, etc. are given below.

$$
\begin{aligned}
& C_{1}=\frac{0.034(n-1)^{2}\left(1-4 v+4 v^{2}\right)}{(-\ln \rho)^{2} \psi^{2}} \\
& C_{2}=-\frac{0.18(n-1)^{5 / 2}\left(1-4 v+4 v^{2}\right) \phi^{1 / 2}}{(-\ln \rho)^{3 / 2} \psi^{2}} \\
& C_{3}=-\frac{(n-1)^{4}}{116.64} \\
& C_{4}=\frac{0.024(n-1)^{3}\left(1-4 v+4 v^{2}\right) \phi}{(-\ln \rho) \psi^{2}} \\
& C_{5}=\frac{0.12(n-1)\left(2-5 v+2 v^{2}\right)}{(-\ln \rho) \psi}
\end{aligned}
$$




$$
\begin{aligned}
& C_{6}=-\frac{0.32(n-1)^{3 / 2}\left(2-5 v+2 v^{2}\right) \phi^{1 / 2}}{(-\ln \rho)^{1 / 2} \psi} \\
& C_{7}=0.035\left(16-4 v+7 v^{2}\right) .
\end{aligned}
$$

Solution of equation (21) for interference, $\delta_{n}^{*}$, by substituting $\Delta T_{n}^{*}=\Delta T_{(s l i)_{n}}^{*}$ from equation (19), gives the apparent critical values of interference $\left(\delta_{(s a)_{n}}^{*}\right)$ and curvature coefficient $\left(c_{(s a)_{n}}\right)$ at which slipping of an n-point asperity before yielding occurs. All n-point asperities having interference $\delta_{n}^{*}<\delta_{(s a)_{n}}^{*}$ will contribute to tangential slip resistance. But there exists some asperities which do not slip and still resist tangential force until their yielding inception. The frictional or tangential force contributed by such n-point asperities which lie in the interference range $\delta_{(s a)_{n}}^{*} \leq \delta_{n}^{*} \leq \delta_{(y a)_{n}}^{*}$ is obtained by replacing $\Delta T_{n}^{*}$ by $\Delta T_{(y i e l d)_{n}}^{*}$ in equation (21) and solving it for $\Delta T_{(\text {yield })_{n}}^{*}$. This solution gives two roots for $\Delta T_{(\text {yield })_{n}}^{*}$ as given below.

$$
\Delta T_{(\text {yield })_{n}}^{*}=\frac{-\left(\text { Term } I \pm(\text { Term II })^{I / 2}+\text { Term III }\right)}{\operatorname{Term} I V}
$$

$\operatorname{Term} I=C_{6} c_{n}^{1 / 4} \delta_{n}^{* 3 / 4}$

$$
\begin{aligned}
\text { Term II }= & \left(C_{6}^{2} c_{n}^{1 / 2} \delta_{n}^{* 3 / 2}+C_{5}^{2} c_{n} \delta_{n}^{* 3}-4 C_{3} C_{7} \delta_{n}^{* 2}-4 C_{1} C_{7} c_{n} \delta_{n}^{* 3}-4 C_{2} C_{7} c_{n}^{3 / 4} \delta_{n}^{* 9 / 4}-4 C_{4} C_{7} c_{n}^{1 / 2} \delta_{n}^{* 3 / 2}\right. \\
& \left.+2 C_{5} C_{6} c_{n}^{3 / 4} \delta_{n}^{* 9 / 4}\right)^{\frac{1}{2}}
\end{aligned}
$$

Term III $=C_{5} c_{n}^{I / 2} \delta_{n}^{* 3 / 2}$

Term $\mathrm{IV}=2 \mathrm{C}_{7} c_{n}$

\section{Modeling Adhesive Frictional Contact: KE Approach}

As compared to the analysis given above, the present section differs only in the way of treating the intermediate elastic-plastic transition zone. In this section instead of CEB's analytical approach expressions, KE finite element approach expressions are used. While modeling elasticplastic contact of a single asperity, Kogut and Etsion [13] found that the analytical approaches like CEB gives discontinuity in load-deformation behaviour at elastic to plastic transition point but FEA (Finite element analysis) approaches accurately predict such behaviour at transition. Based upon their accurate FEA results for elastic-plastic contact of single asperity, they developed expressions for transition zone behaviour of load and contact area. Same expressions are incorporated in present section to model elastic-plastic adhesive contact using n-point asperities. In earlier study also the present authors (Waghmare and Sahoo, [22]) have successfully incorporated the KE expressions for non-adhesive frictional contact. The present adhesive friction contact study is based upon RG's slip and yield theory of contacting asperities. As compared to existing RG model of adhesive frictional contact, the novelty of present section work lies in use of KE approach expressions for predicting normal and tangential load in elastic-plastic transition zone and modeling the contact problem in npoint asperity frame work.

\section{Applied Load Formulation for Single n-Point Asperity}

KE model considers four distinct zones of an asperity deformation in elastic-plastic contact progression. The same four zones are used in present section study of elastic-plastic adhesive contact progression with an n-point asperity. In terms of critical value of interference $\left(\delta_{(y)_{n}}\right)$, marking yield inception, these zones of deformation are as given below. 
$0<\delta_{n}<\delta_{(y)_{n}}$ Predominantly elastic zone

$\delta_{(y)_{n}} \leq \delta_{n} \leq 6 \delta_{(y)_{n}}$ Predominantly elastic-plastic stage-I zone

$6 \delta_{(y)_{n}}<\delta_{n} \leq 110 \delta_{(y)_{n}}$ Predominantly elastic-plastic stage-II zone

$\delta_{n}>110 \delta_{(y)_{n}}$ Predominantly plastic zone

Now, for contact between single n-point asperity and a rigid flat plane, four distinct phases of deformation, can be modeled as discussed below.

For purely elastic and purely plastic deformation of an n-point asperity, the same theories as discussed in above section are used (i.e. JKR theory [27] for elastic deformation and Roy Chowdhury and Pollock theory [28] for plastic deformation). For purely elastic part of formulation, the same equations viz. (17) is used. But for purely plastic part of formulation, the equation (18) as give in above section needs to be modified. This is because, in above section, CEB approach of conservation of asperity tip volume is used to calculate the contact area, while in this section as KE approach is being used therefore the contact area in purely plastic deformation needs to be calculated by using truncation volume concept. So, corresponding changes are incorporated in equation (18) to get applied load value of purely plastic deformation. With these considerations, load required to be applied for elastic and plastic type of deformation in presence of adhesion can be given as.

$$
\begin{aligned}
& \Delta P_{(e)_{n}}^{*}=\frac{(n-1)}{(-\ln \rho)}\left\{\frac{0.67 \delta_{n}^{* 3 / 2}}{\psi c_{n}^{1 / 2}-\frac{1.77(n-1)^{1 / 2}(-\ln \rho)^{1 / 2} \phi^{1 / 2} \delta_{n}^{* 3 / 4}}{\psi c_{n}^{3 / 4}}}\right\} \\
& \text { for } \delta_{(0)_{n}}^{*}<\delta_{n}^{*}<\delta_{(y a)_{n}}^{*} \\
& \Delta P_{(p)_{n}}^{*}=1.57(n-1)^{2}\left(\frac{\delta_{n}^{*}}{c_{n}}-\frac{\phi}{c_{n} \psi}\right) \quad \text { for } \delta_{n}^{*}>110 \delta_{(y)_{n}}^{*}
\end{aligned}
$$

All parameters used in above two equations have same meaning as given in above section.

As per KE model, the total elastic-plastic transition zone extends from yielding inception point to fully plastic flow point and these points are defined in terms of critical values of asperity interference. The critical interference $\delta_{(y)_{n}}$, marks the yielding inception and it is determined by following Tabor [29] criteria, while $110 \delta_{(y)_{n}}$, marks the fully plastic flow. This total transition zone which lies between $\delta_{(y)_{n}}$ and $110 \delta_{(y)_{n}}$ has two sub regions with transition at interference value of $\delta_{n}=6 \delta_{(y)_{n}}$. KE model analytical expressions which predict load-deformation behaviour in transition zone are given as

$$
\begin{aligned}
& \Delta P_{(e p-I)_{n}}=1.03 \Delta P_{(y)_{n}}\left(\frac{\delta_{n}}{\delta_{(y)_{n}}}\right)^{1.425} \text { for } \delta_{(y)_{n}} \leq \delta_{n} \leq 6 \delta_{(y)_{n}} \\
& \Delta P_{(e p-I I)_{n}}=1.40 \Delta P_{(y)_{n}}\left(\frac{\delta_{n}}{\delta_{(y)_{n}}}\right)^{1.263} \text { for } 6 \delta_{(y)_{n}}<\delta_{n} \leq 110 \delta_{(y)_{n}}
\end{aligned}
$$

where $\Delta P_{(y)_{n}}$ is the critical value (value corresponding to the point where elastic to elasto-plastic transition takes place) of applied load on a single n-point asperity for a specific value of ' $n$ ' and is obtained by solving equation (23) used for purely elastic regime and by assigning interference value as $\delta_{n}=\delta_{(y)_{n}}$. As equation (23) is obtained by applying JKR theory of adhesion, therefore we can say that the transition zone behaviour gets indirectly governed by this theory of adhesion. As it is a 
parametric study therefore to get generalized solution, all load values are normalized by dividing with load dimension term $\left(A_{0} H\right)$, which is product of nominal contact area and hardness of asperity material. Load values in equations (23) and (24) are already in normalized form. Equations (25) and (26) in normalized form are as given below.

$$
\begin{aligned}
& \Delta P_{(e p-I)_{n}}^{*}=\left\{\frac{0.49(n-1) \delta_{n}^{* 1.425}}{(-\ln \rho) \psi \delta_{(y a)_{n}}^{* 0.425}}-\frac{1.08(n-1)^{3 / 2} \phi^{1 / 2} \delta_{n}^{*^{1.425}}}{(-\ln \rho)^{1 / 2} \psi \delta_{(y a)_{n}}^{* 1.425}}\right\} \quad \text { for } \delta_{(y a)_{n}}^{*}<\delta_{n}^{*} \leq 6 \delta_{(y a)_{n}}^{*} \\
& \Delta P_{(e p-I I)_{n}}^{*}=\left\{\frac{0.66(n-1) \delta_{n}^{* 1.263}}{(-\ln \rho) \psi \delta_{(y a)_{n}}^{* 0.263}}-\frac{1.47(n-1)^{3 / 2} \phi^{1 / 2} \delta_{n}^{* 1.263}}{(-\ln \rho)^{1 / 2} \psi \delta_{(y a)_{n}}^{*^{1.263}}}\right\} \quad \text { for } 6 \delta_{(y a)_{n}}^{*}<\delta_{n}^{*} \leq 110 \delta_{(y a)_{n}}^{*}
\end{aligned}
$$

It is to be noted that the plasticity index $(\psi)$ [18] and adhesion index $(\phi)$ [30], got introduced in above equations (23) - (24) and (27) - (28) in normalization process.

\section{Frictional Load Formulation for Single n-Point Asperity}

KE demarcated four distinct zones of asperity deformation, viz. elastic, elastic-plastic stage-I (predominantly elastic), elastic-plastic stage-II (predominantly plastic), and plastic. Now, for modeling the frictional load, first it is necessary to fix which sorts of asperities in each zone of deformation contribute to it. Kogut and Etsion [32] in their frictional contact study have proved that the contacting asperities only in elastic and predominantly elastic zone of contact (elastic-plastic stage-I) support tangential load. So accordingly in present study to calculate tangential load values corresponding to slip and yield points, the limits of asperity deformation are modified. But the modeling procedure for slip and yield parts of frictional load are the same as given in above section. The slipping of the asperities because of tangential load may occur either in purely elastic or elasticplastic stage-I zone of deformation. Therefore, resistance offered by such asperities before slip is calculated as frictional force using equation (19) while the resistance offered by asperities which do not slip is calculated by equation (22).

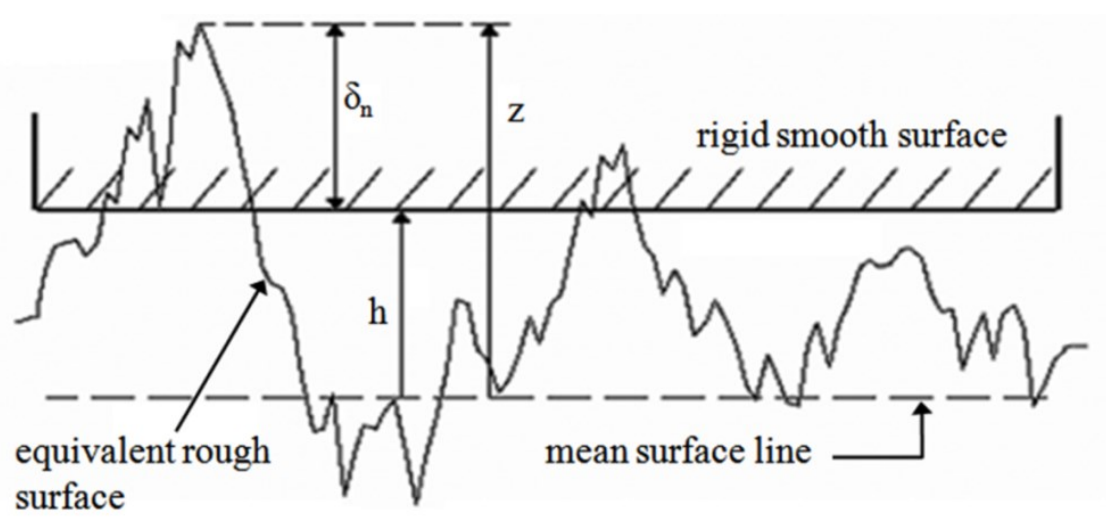

Figure 4. Contact of equivalent rough surface and rigid smooth surface.

\section{Modeling Rough Surface Contact}

After modeling applied load and frictional load for single n-point asperity as given above, the probabilistic multi asperity approach is adopted to model the behaviour of nominally flat contacting surfaces. The contact between two rough surfaces is first simplified in to an equivalent situation of contact between equivalent rough surface and a rigid flat surface (see Fig. 4) (Greenwood and Tripp [33]; O'Callaghan and Cameron [34]). All the asperities emerging because of interference between a rigid flat plane and the equivalent rough surface are treated as n-point asperities. The statistical existence of each n-point asperity is evaluated by using equation (1) with necessary changes in it to accommodate the equivalent asperity parameters. Variation in size of an n-point asperity is achieved 
by using a Gaussian joint probability density function (pdf) combined with exponential auto correlation function as given in equation (2). The number of n-point asperities emerging out of interference will be the product of total number of sample points (height ordinates) considered on the sample space of surface under study and the probability of existence of each asperity.

\section{Applied Load on Rough Surface: CEB Approach}

In CEB approach the different zones of deformation are marked as given below.

$$
\begin{array}{ll}
\delta_{(0)_{n}}^{*} \leq \delta_{n}^{*} \leq \delta_{(y a)_{n}}^{*} & \text { purely elastic deformation regime. } \\
\delta_{(y)_{n}}^{*} \leq \delta_{n}^{*} \leq \infty & \text { elasto-plastic and purely plastic regimes of deformation. }
\end{array}
$$

If there exist $M_{(e)_{n}}$ and $M_{(p)_{n}}$ number of n-point asperities in the above two regimes respectively then for a sample space of $M$ number of height ordinates,

$$
\begin{array}{ll}
M_{(e)_{n}}=M \times N_{(e)_{n}} & \text { for } \delta_{(0)_{n}}^{*} \leq \delta_{n}^{*} \leq \delta_{(y a)_{n}}^{*} \\
M_{(p)_{n}}=M \times N_{(p)_{n}} & \text { for } \delta_{(y)_{n}}^{*} \leq \delta_{n}^{*} \leq \infty
\end{array}
$$

where $N_{(e)_{n}}$ and $N_{(p)_{n}}$ give probability of existence of single n-point asperity in above two respective zones and are evaluated by using equation (1) for the corresponding limits of interference of an equivalent n-point asperity. The applied load for these groups of n-point asperities is calculated by taking product of number of asperities in contact and the respective expected value for single n-point asperity.

$$
\begin{array}{ll}
P_{(e)_{n}}^{*}=M_{(e)_{n}} E\left\lfloor\Delta P_{(e)_{n}}^{*}\right\rfloor & \text { for } \delta_{(0)_{n}}^{*} \leq \delta_{n}^{*} \leq \delta_{(y a)_{n}}^{*} \\
P_{(p)_{n}}^{*}=M_{(p)_{n}} E\left\lfloor\Delta P_{(p)_{n}}^{*}\right\rfloor & \text { for } \delta_{(y)_{n}}^{*} \leq \delta_{n}^{*} \leq \infty
\end{array}
$$

The expectation operator $E[$.$] is used to symbolically represent the mathematical procedure followed$ to calculate average or mean value of a random variable in defined range of variation and with defined pattern of distribution of the random variable in that range.

As can be seen in equation (31), the load calculations in elastic domain of contact are based upon apparent limits of interference. So to get apparent interference corresponding to actual interference of zero, one needs to put $\delta_{n}^{*}=0, \delta_{(a)_{n}}^{*}=\delta_{(0)_{n}}^{*}$, and $c_{(a)_{n}}=2 \delta_{(0)_{n}}^{*}$ in above equation (13), that yields,

$$
\delta_{(0)_{n}}^{*}=\{1.22(n-1)(-\ln \rho) \phi\}^{1 / 2}
$$

Load on all asperities having specific $n$ value and deforming in elastic and plastic domain of contact is

$$
P_{n}^{*}=P_{(e)_{n}}^{*}+P_{(p)_{n}}^{*}
$$

Total applied load on the rough surface under consideration is the summation of all $P_{n}^{*}$ calculated for all $n$ values and it is,

$$
P^{*}=\sum_{n=3}^{\infty} P_{n}^{*}
$$

\section{Frictional Force on Rough Surface: CEB Approach}

The formulation of frictional load for single n-point asperity as given above in section is incorporated into a probabilistic multi-asperity contact model by following the same methodology 
as narrated above. Accordingly, the non dimensional frictional force contributed by all n-point asperities with specific ' $n$ ' value (i.e. all those n-point asperities which are comprised of specific ' $n$ ' number of ordinates), before slip and before yield can be calculated as given below.

If there exist $M_{(s i p-e)_{n}}$ number of asperities which support the tangential load before slip in elastic and elastic-plastic stage-I zone of deformation, $M_{(y i e l d-e)_{n}}$ number of asperities which support the tangential load without slip in elastic and elastic-plastic stage-I zone of deformation, then

$$
\begin{gathered}
M_{(s l i p-e)_{n}}=M \times N_{(s l i p-e)_{n}} \quad \text { for } \quad \delta_{(0)_{n}}^{*} \leq \delta_{n}^{*} \leq \delta_{(s a)_{n}}^{*} \\
M_{(\text {yield-e })_{n}}=M \times N_{(\text {yield-e })_{n}} \quad \text { for } \quad \delta_{(s a)_{n}}^{*} \leq \delta_{n}^{*} \leq \delta_{(y a)_{n}}^{*}
\end{gathered}
$$

where $N_{(s l i p-e)_{n}}$ and $N_{(y i e l d-e)_{n}}$ give probability of existence of single n-point asperity in above two respective zones and are evaluated by using equation (1) for the corresponding limits of interference of an equivalent n-point asperity.

Now, the tangential load supported by such n-point asperities on the surface which are all comprised of specific ' $n$ ' number of height ordinates will be

$$
\begin{aligned}
& T_{(s l i p)_{n}}^{*}=M_{(s l i p-e)_{n}} E\left\lfloor\Delta T_{(s l i p)_{n}}^{*}\right\rfloor \quad \text { for } \quad \delta_{(0)_{n}}^{*} \leq \delta_{n}^{*} \leq \delta_{(s a)_{n}}^{*} \\
& T_{\left({\text {yield })_{n}}_{n}\right.}^{*}=M_{(\text {yield-e) })_{n}} E\left[\Delta T_{(\text {yield })_{n}}^{*}\right] \quad \text { for } \quad \delta_{(s a)_{n}}^{*} \leq \delta_{n}^{*} \leq \delta_{(y a)_{n}}^{*}
\end{aligned}
$$

Total frictional force contributed by all n-point asperities with specific ' $n$ ' value (i.e. which are all comprised of specific ' $n$ ' number of ordinates) is

$$
T_{n}^{*}=T_{(s i p)_{n}}^{*}+T_{(y i e l d)_{n}}^{*}
$$

Total frictional force on the whole surface in contact is

$$
T^{*}=\sum_{i=3}^{n} T_{n}^{*}
$$

\section{Applied Load on Rough Surface: KE Approach}

If probability of occurrence of single n-point asperity in four zones of deformation are designated as - $N_{(e)_{n}}$ for elastic zone, $N_{(e p-I)_{n}}$ for elastic-plastic state-I zone, $N_{(e p-I)_{n}}$ for elasticplastic state-II zone, and $N_{(p)_{n}}$ for plastic zone, then the number of n-point asperities in contact in each of these zones can be calculated as given below.

$$
\begin{array}{lll}
M_{(e)_{n}}=M \times N_{(e)_{n}} & \text { for } & \delta_{\left(()_{n}\right.}^{*}<\delta_{n}^{*}<\delta_{(y a)_{n}}^{*} \\
M_{(e p-I)_{n}}=M \times N_{(e p-I)_{n}} & \text { for } & \delta_{(y a)_{n}}^{*} \leq \delta_{n}^{*}<6 \delta_{(y a)_{n}}^{*} \\
M_{(e p-I I)_{n}}=M \times N_{(e p-I I)_{n}} & \text { for } & 6 \delta_{(y a)_{n}}^{*}<\delta_{n}^{*} \leq 110 \delta_{(y a)_{n}}^{*} \\
M_{(p)_{n}}=M \times N_{(p)_{n}} & \text { for } & \delta_{n}^{*}>110 \delta_{(y)_{n}}^{*}
\end{array}
$$

The applied load for group of n-point asperities which are all comprised of same ' $n$ ' number of height ordinates and undergoing deformation of particular type is calculated by taking product of number of asperities in contact and the respective expected value for single n-point asperity.

$$
\begin{array}{ll}
P_{(e)_{n}}^{*}=M_{(e)_{n}} E\left\lfloor\Delta P_{(e)_{n}}^{*}\right\rfloor & \text { for } \delta_{(0)_{n}}^{*}<\delta_{n}^{*}<\delta_{(y a)_{n}}^{*} \\
P_{(e p-I)_{n}}^{*}=M_{(e p-I)_{n}} E\left\lfloor\Delta P_{(e p-I)_{n}}^{*}\right\rfloor & \text { for } \delta_{(y a)_{n}}^{*} \leq \delta_{n}^{*} \leq 6 \delta_{(y a)_{n}}^{*}
\end{array}
$$




$$
\begin{array}{ll}
P_{(e p-I I)_{n}}^{*}=M_{(e p-I)_{n}} E\left\lfloor\Delta P_{(e p-I I)_{n}}^{*}\right\rfloor & \text { for } 6 \delta_{(y a)_{n}}^{*}<\delta_{n}^{*} \leq 110 \delta_{(y a)_{n}}^{*} \\
P_{(p)_{n}}^{*}=M_{(p)_{n}} E\left\lfloor\Delta P_{(p)_{n}}^{*}\right\rfloor & \text { for } \delta_{n}^{*}>110 \delta_{(y)_{n}}^{*}
\end{array}
$$

The expectation operator $E[$.$] , gives expected values of applied loads in respective range of$ deformation for single n-point asperity.

Total applied load for asperities having specific ' $n$ ' value is,

$$
P_{n}^{*}=P_{(e)_{n}}^{*}+P_{(e p-I)_{n}}^{*}+P_{(e p-I I)_{n}}^{*}+P_{(p)_{n}}^{*}
$$

Total applied load due to all asperities on rough surface under consideration is,

$$
P^{*}=\sum_{n=3}^{\infty} P_{n}^{*}
$$

\section{Frictional Force on Rough Surface: KE Approach}

If there exist $M_{(s i p-e)_{n}}$ and $M_{(s i p-e p-I)_{n}}$ number of asperities which support the tangential load before slip in elastic and elastic-plastic stage-I zone of deformation respectively, $M_{(y i e l d-e)_{n}}$ and $M_{(y i e l d-e p-I)_{n}}$ number of asperities which support the tangential load without slip in elastic and elastic-plastic stage-I zone of deformation respectively, then

$$
\begin{aligned}
& M_{(s l i p-e)_{n}}=M \times N_{(s l i p-e)_{n}} \\
& \left.\begin{array}{r}
\text { for } \delta_{(0)_{n}}^{*}<\delta_{n}^{*}<\delta_{(s a)_{n}}^{*} \\
\text { and } \delta_{(s a)_{n}}^{*}<\delta_{(y a)_{n}}^{*}
\end{array}\right\} \\
& M_{(s l i p-e p-I)_{n}}=M \times N_{(s l i p-e p-I)_{n}} \\
& \left.\begin{array}{c}
\text { for } \quad \delta_{(y a)_{n}}^{*} \leq \delta_{n}^{*}<\delta_{(s a)_{n}}^{*} \\
\text { and } \delta_{(y a)_{n}}^{*} \leq \delta_{(s a)_{n}}^{*} \leq 6 \delta_{(y a)_{n}}^{*}
\end{array}\right\} \\
& M_{(y i e l d-e)_{n}}=M \times N_{(y i e l d-e)_{n}} \\
& \left.\begin{array}{rl}
\text { for } & \delta_{(s a)_{n}}^{*} \leq \delta_{n}^{*} \leq \delta_{(y a)_{n}}^{*} \\
& \text { and } \quad \delta_{(s a)_{n}}^{*}<\delta_{(y a)_{n}}^{*}
\end{array}\right\} \\
& \left.\begin{array}{l}
\text { for } \quad \delta_{(s a)_{n}}^{*} \leq \delta_{n}^{*} \leq 6 \delta_{(y a)_{n}}^{*} \\
\text { and } \quad \delta_{(y a)_{n}}^{*} \leq \delta_{(s a)_{n}}^{*} \leq 6 \delta_{(y a)_{n}}^{*}
\end{array}\right\}
\end{aligned}
$$

Now, the tangential load supported by such n-point asperities on the surface which are all comprised of specific ' $n$ ' number of height ordinates will be

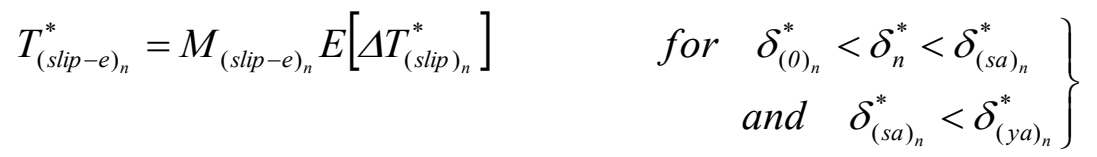

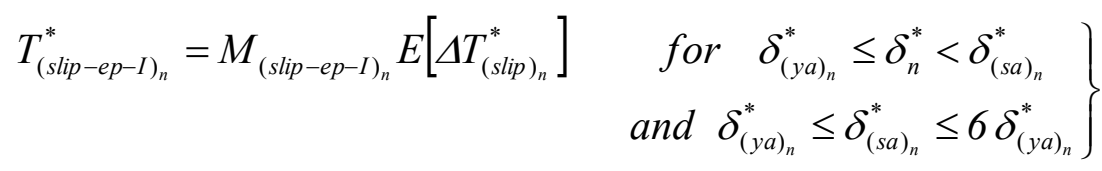

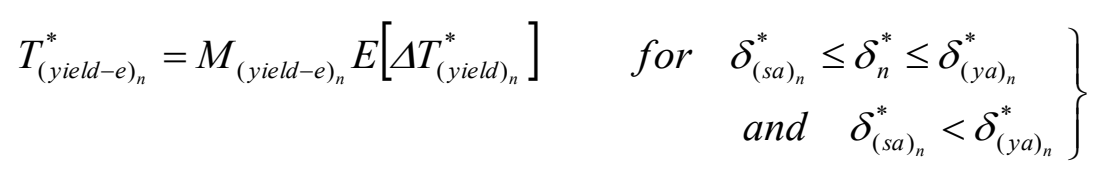

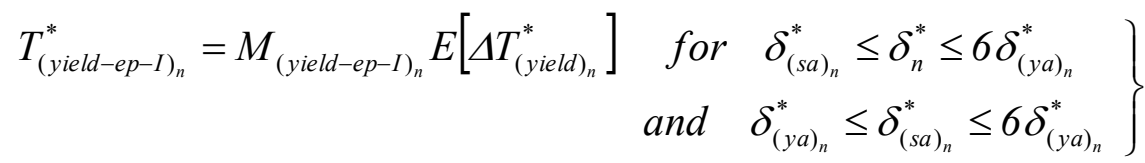


As compared to equations (38) and (39) which are used for CEB approach, above equations (56)-(59) used for KE approach differ in limits of interference values. The slip point interference $\delta_{(s a)_{n}}^{*}$ considered in CEB approach [equation (38)] lies in purely elastic zone while that in KE approach may lie either in purely elastic [equation (56)] or elastic-plastic stage-I [equation (57)] zone. Also to calculate tangential load sustained by asperities which do not slip is calculated by considering asperities in elastic [equation (58)] and elastic-plastic stage-I [equation (59)] zone in $\mathrm{KE}$ approach, while in CEB approach only the asperities in elastic zone are considered to get this value [equation (39)].

Total frictional force contributed by all n-point asperities with specific ' $n$ ' value (i.e. which are all comprised of specific ' $n$ ' number of ordinates) is

$$
T_{n}^{*}=T_{(s l i p-e)_{n}}^{*}+T_{(s l i p-e p-I)_{n}}^{*}+T_{(y i e l d-e)_{n}}^{*}+T_{(y i e l d-e p-I)_{n}}^{*}
$$

Total frictional force on the whole surface in contact is

$$
T^{*}=\sum_{i=3}^{n} T_{n}^{*}
$$

\section{Results and Discussion}

In order to study adhesive friction with two different approaches viz., CEB and KE, the corresponding formulations as modeled above for total applied load and total frictional force are solved by using MATLAB code. The applied load and tangential load formulations consists of different surface roughness and material properties related parameters like number of height ordinates $(n)$, correlation coefficient $(\rho)$, adhesion index $(\phi)$ and plasticity index $(\psi)$. The different conditions of contact with respect to variation in material properties and surface roughness features are obtained by varying the well-defined indices, viz., the plasticity index $(\psi)$ [18] and adhesion index $(\phi)$ [30]. Care is taken in selection of these indices for study so that we get significantly different zones with respect to plasticity effect and adhesion effect. For the fixed material conditions and surface roughness values (i.e constant $(\phi$ and $\psi)$ variation in applied normal load and frictional load values are obtained by varying the mean surface separation $\left(h^{*}=h / \sigma\right)$ between contacting surfaces in the range of 0 to. $+3 \sigma$. For standard normal distribution of height ordinates, this range of mean separation covers $99.7 \%$ asperities on the surface under consideration. Physical significance of the different parameters and the related data used in present study is as discussed below.

Physically, correlation length $(\beta)$ gives an indication of the nature of the profile or surface. It represents the maximum distance over which significant correlation in roughness heights exist. In other words, correlation length is the distance between two statistically independent points. Smoother surfaces generally have large correlation lengths while rougher surfaces have low values of correlation lengths. A profile of white noise (representing perfectly rough surface) have zero correlation length while a straight line profile (representing perfectly smooth surface) have correlation length equal to infinity. Standard deviation of asperity heights $(\sigma)$ (RMS height) and correlation length $(\beta)$ gives roughness measure on vertical and horizontal scale respectively. Thus we can say that the roughness of surface corresponds to high values of RMS heights and low values of correlation lengths. A surface profile with low value of correlation length will carry thin and sharp asperities while that having high value of correlation length will have thick and flat asperities. In the present study practical value of correlation length $(\beta)$ is chosen as $6.5 \mu \mathrm{m}$ which is same as that used in earlier studies [18, 30].

Correlation coefficient $(\rho)$ defines the extent of similarity between the asperities and it carries values in between 0 and 1 . For high correlation coefficient, the surface will carry highly correlated 
or highly similar asperities, and vice versa. As two extremities of similarity are impracticable, therefore an average value i.e. $\rho=0.5$ is used as correlation coefficient in present study. It is to be noted that the correlation coefficient $(\rho)$ may vary by changing the sampling length $(\Delta x)$. This is because for a surface on which sampled points are distributed with exponential type of auto correlation function, the sampling length, correlation length, and correlation coefficient are related as, $\Delta x=\beta(-\ln \rho)$, and correlation length $(\beta)$ being a characteristic of surface under study, it remains constant. The choices of $\rho$ and $n_{\max }$ (maximum value of $n$ or maximum value of the number of height ordinates of which an asperity is comprised of) are dependent on each other. The value of $\rho$ dictates the choice of $n_{\max }$ to a large extent. Hariri et al. [17] have shown that for low value of $\rho$, lower value of $n_{\max }$ is sufficient and for high value of $\rho$, higher value of $n_{\max }$ is required. Earlier studies of Hariri et al. [18] and Sahoo et al. [30] have shown that with $\rho=0.67$ and $n_{\max }=7$ accurate results can be obtained down to $h=0$. In the present work also asperities with maximum up to 7 number of ordinates $\left(n_{\max }=7\right)$ are considered but with $\rho=0.5$. This selected value of correlation coefficient represents a compromise value between the maximum and minimum values of correlation. Though it is better to consider higher $n_{\max }$ for a particular value of $\rho$ but it increases the numerical computational complexity with almost negligible improvement in accuracy. In present work, the non dimensional separation $(h / \sigma)$ between rigid smooth surface and equivalent rough surface is considered between 0 to +3 and for Gaussian distribution of asperity heights this range of separation covers almost $100 \%$ of the asperities.

The plasticity index $(\psi=(H B) /(E \sigma))$, is function of hardness $(H)$ of the softer of two contacting surfaces, composite elastic modulus $(E)$ of contacting surfaces, and surface roughness parameters $(\beta$ and $\sigma)$. The plasticity index is responsible for creation of prospective contact situations due to variation in applied load, material properties and surface roughness. Higher the value of plasticity index $(\psi)$, lower will be plasticity effect, and vice versa. Plasticity index values help to demarcate the whole regime of contact deformation for predominance of particular type of deformation. In present study three different values of $\psi$ are used, viz., 2.0, 0.9, and 0.3 to represent predominantly elastic, elasto-plastic, and plastic regimes of asperity deformation respectively.

This adhesion index $(\phi)$, which is same as that defined in Sahoo et al. [30] study, combines the work of adhesion per unit contact area $(\gamma)$, composite elastic modulus of contacting surfaces ( $E)$ and surface roughness parameters $(\beta$ and $\sigma)$. Higher the values of $\phi$, higher will be adhesion effect and vice versa. Earlier analysis [30] considered the adhesion index in the range 0.1 to 0.9 . As per Israelachvili [35], many materials have surface energies of the order $0.01-0.05 \mathrm{~J} / \mathrm{m}^{2}$ and metals typically have higher values of the order $0.4-4 \mathrm{~J} / \mathrm{m}^{2}$. The interfacial energies are of the same order of magnitude as surface energies. The work of adhesion for two surfaces in contact is obtained by combining the surface energies of individual surfaces and the energy of interfacial surface. Typical values of root mean square value of surface roughness $(\sigma)$ for nano to micro scale surfaces are in the range of $59 \mathrm{~nm}$ to $0.3 \mu \mathrm{m}$ and correlation length, $\beta=6.5 \mu \mathrm{m}$ (Whitehouse and Archard [36]). For metals like steel, the average value of elastic modulus is $210 \mathrm{GPa}$ and Poisson's ratio is 0.29. With this the reduced or equivalent modulus of elasticity works out as $114 \mathrm{GPa}$. Thus with realistic estimate of surface roughness values and material property values as mentioned, the adhesion index $(\phi)$ lies in the range of 0.01 to 0.9 . The three different adhesion index values used in present study, viz., $\phi=0.1,0.5$, and 0.9 , cover low, moderate, and high adhesion effect zones respectively.

First the results are obtained by solving CEB approach formulations for total applied load and total friction force on the rough surface (plots in Fig. 5-8). Plots in Fig. 5 (a, b, c) and Fig. 6 (a, b, c) show variation in non-dimensional friction force with variation in non-dimensional mean separation between contacting surfaces. The general trend of behaviour in all the plots is with decrease in distance of separation, friction force increases. This can be attributed to the fact that 
with decrease in separation, more number of asperities comes in contact, also the size (radius of curvature) of asperities which are already in contact increases, and cumulative effect of this gives increased friction force.

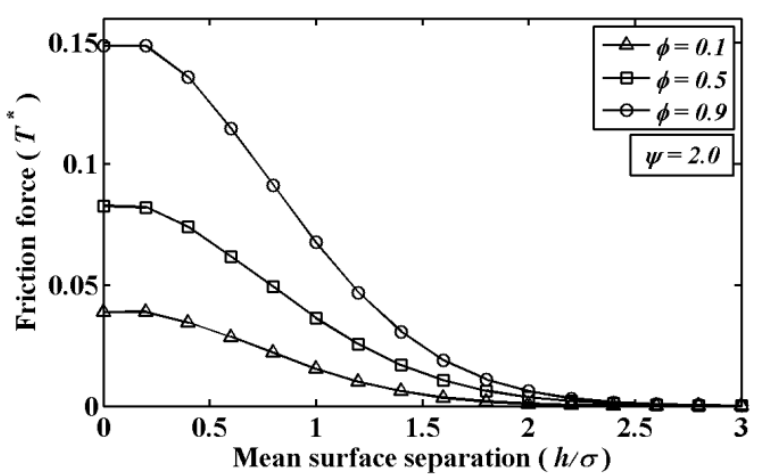

(a)

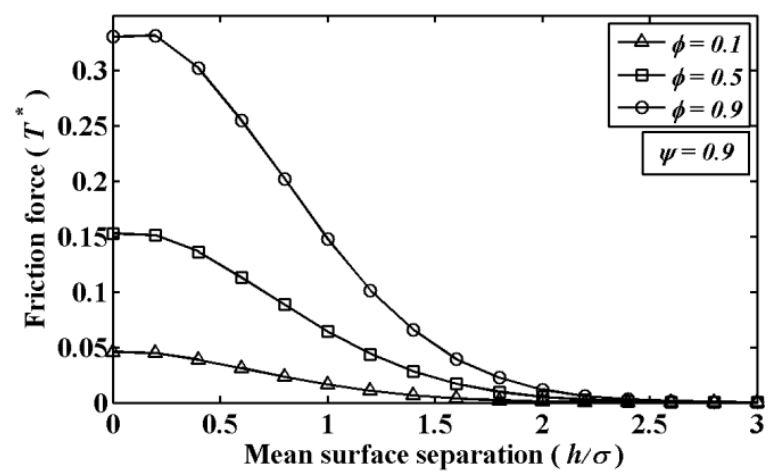

(b)

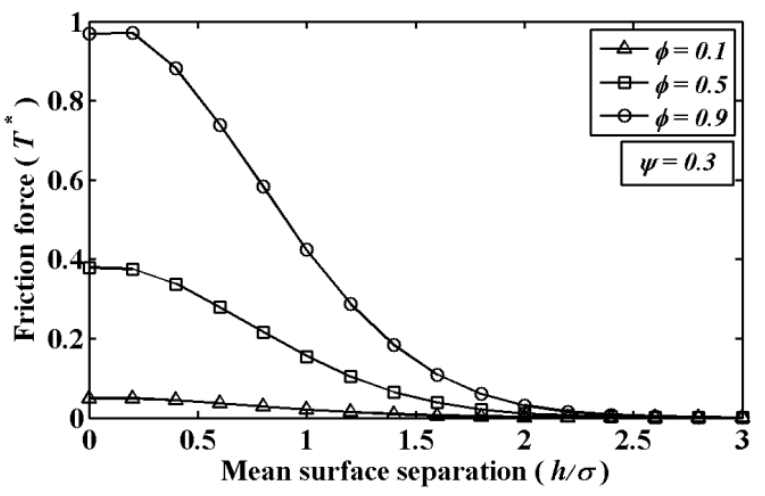

(c)

Figure 5. CEB approach formulation plotted for non-dimensional frictional force versus nondimensional mean separation as a function of adhesion index $(\phi)$ in: (a) predominantly elastic zone $(\psi=2.0)$, (b) predominantly elasto-plastic zone $(\psi=0.9)$, (c) predominantly plastic zone $(\psi=0.3)$.

Plots in Fig. 5 (a, b, c) are function of adhesion index while that in Fig. 6 (a, b, c) are function of plasticity index. Increase in adhesion index value from 0.3 to 0.9 represents increased adhesion effect while decrease in plasticity index from 2.0 to 0.3 represents increased plasticity effect. As can be seen in Fig. 5 (a, b, c), with increase in adhesion effect (i.e. with increase in adhesion index from 0.3 to 0.9 ) friction force at particular level of separation increases. Physically it means higher the adhesion, higher will be bonding (due to secondary forces of attraction) between the contacting asperity surfaces. And in turn it will demand higher tangential forces to shear them. Fig. 6 (a, b, c) show that with increase in plasticity effect (i.e. with decrease in plasticity index from 2.0 to 0.3 ) friction force at particular level of separation increases. Physically it means higher the tendency to deform plastically higher will be frictional force. Rougher surfaces carry slender asperities with higher peaks which have tendency to deform plastically. In short from all plots in Fig. 5 (a, b, c) and Fig. $6(a, b, c)$, it can be concluded that at a particular level of separation between surfaces, friction force increases with increase in adhesion and plasticity effects. Physically, increase in adhesion effect is characteristic of smoother surfaces and/or surfaces possessing higher surface energy, while increase in plasticity effect is the characteristic of rough and/or soft surfaces. 


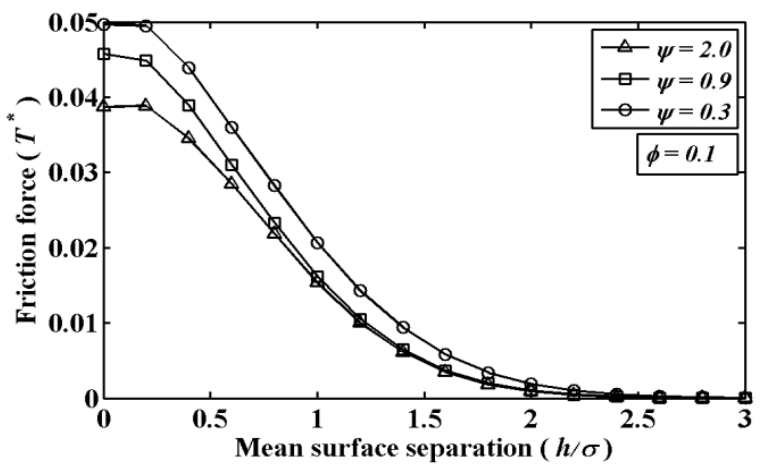

(a)

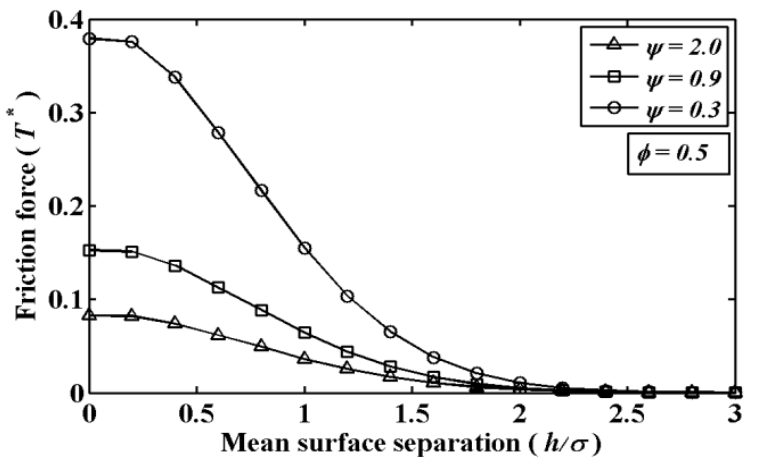

(b)

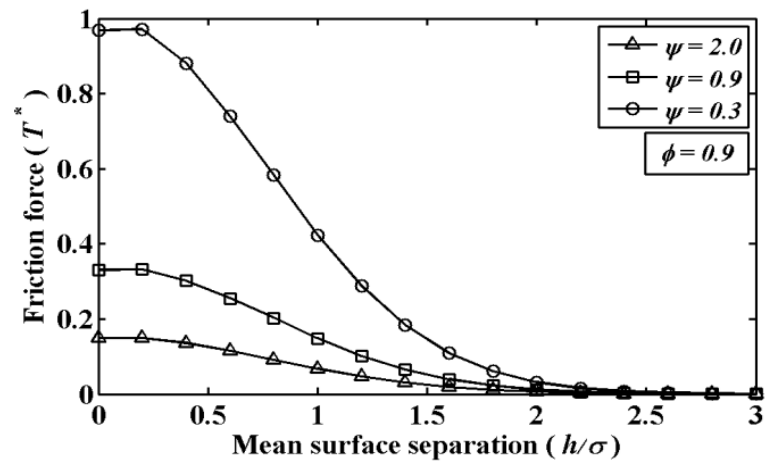

(c)

Figure 6. CEB approach formulation plotted for non-dimensional frictional force versus nondimensional mean separation as a function of plasticity index $(\psi)$ in: (a) low adhesion zone $(\phi=0.1),(\mathrm{b})$ moderate adhesion zone $(\phi=0.5),(\mathrm{c})$ high adhesion zone $(\phi=0.9)$.

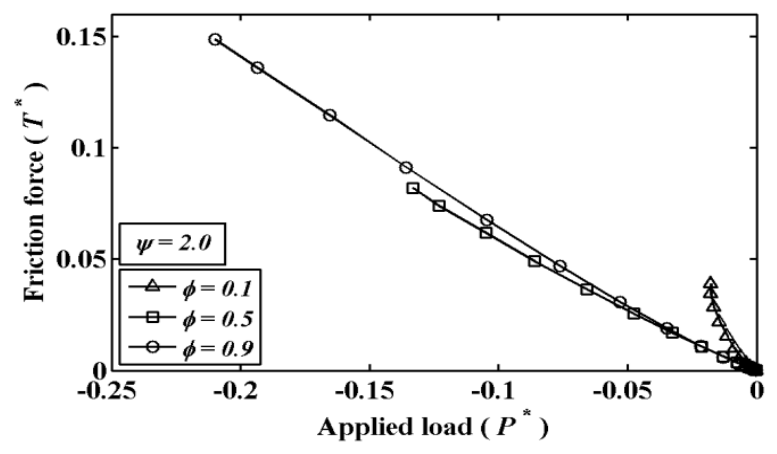

(a)

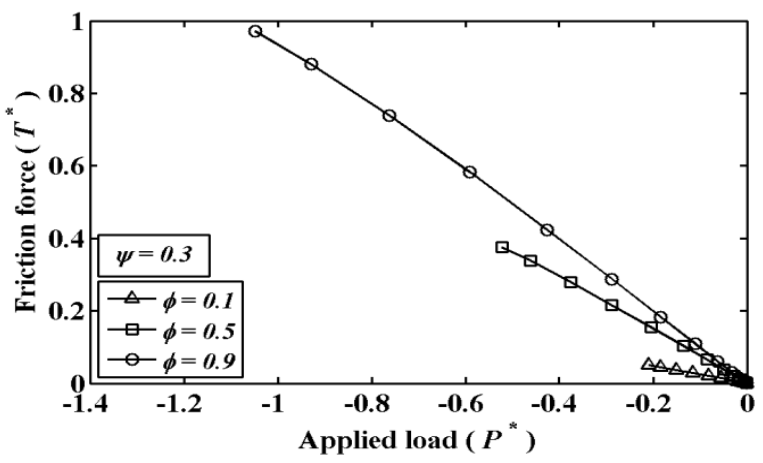

(c)

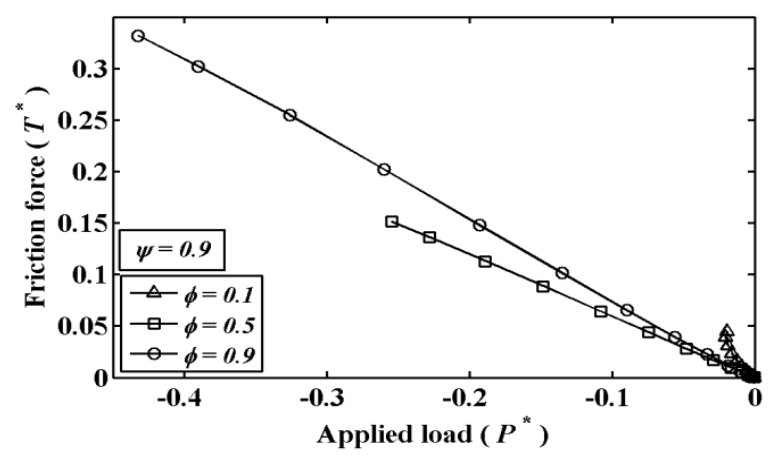

(b)

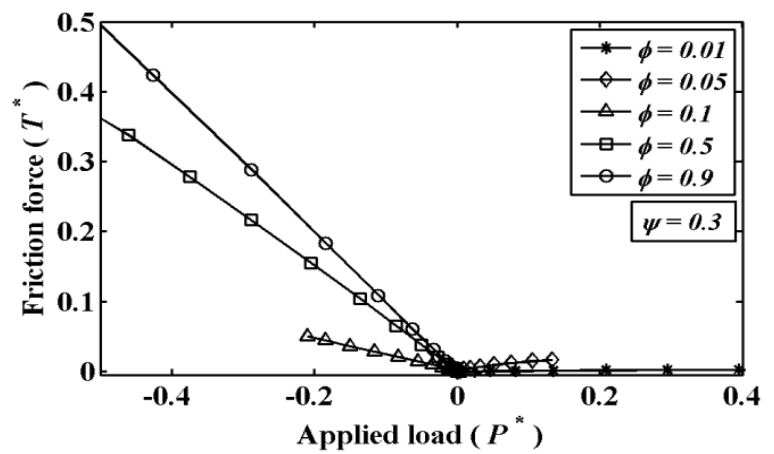

(d)

Figure 7. CEB approach formulation plotted for non-dimensional frictional force versus nondimensional applied load as a function of adhesion index $(\phi)$ in: (a) predominantly elastic zone $(\psi=2.0)$, (b) predominantly elasto-plastic zone $(\psi=0.9)$, (c) predominantly plastic zone $(\psi=0.3)(\mathrm{d})$ predominantly plastic zone $(\psi=0.3)$ and lower values of $\phi$. 
Plots in Fig. 7 (a, b, c) and Fig. 8 (a, b, c) show variation of non-dimensional friction force with variation in non-dimensional normally applied load. The general trend of behaviour in all the plots is that the friction force is almost proportional to normally applied load. In Fig. 7 (a), (b) and Fig. 8 (a), plots with the combination of higher $\psi$ and lower $\phi$ show small non linearity. Physically it means surfaces which have higher tendency to deform elastically and are more rough show nonlinear behaviour of friction force with normally applied load.

From rest of the plots in Fig. 7 and Fig. 8 it is clear that higher the tendency to deform plastically (lower values of $\psi$ ) and/or higher adhesion effect (higher values of $\phi$ ), higher will be linearity in variation of friction load with respect to normally applied load. Fig. 7 (c) and (d) show plots plotted in high zone of plasticity effect (i.e. for low value of $\psi$ ) for different values of $\phi$ representing negligible to high adhesion effect. All these plots are exactly linear and also as can be seen in Fig. 7 (d) negligible adhesion effect values give positive values of applied load. Positive values of applied load indicate predominance of deformation effect forces over adhesion effect forces. In Fig. 7 (c) and (d) it is observed that with the increase in adhesion effect the slope of friction load - normally applied load plots increase in both negative and positive zones of normally applied load.

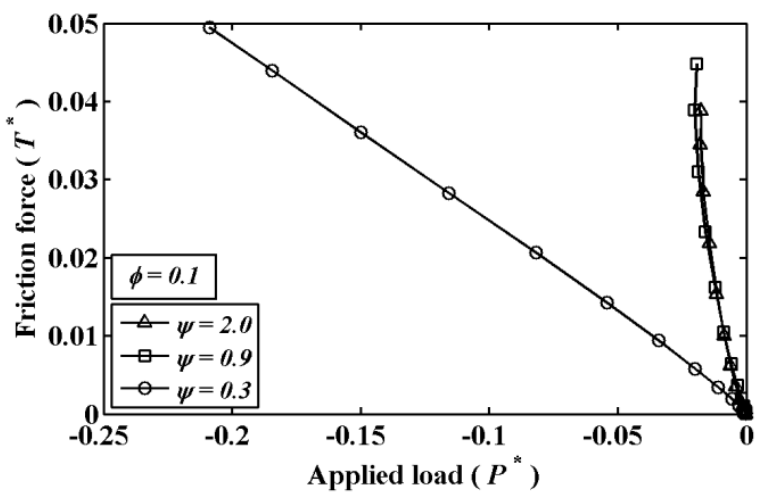

(a)

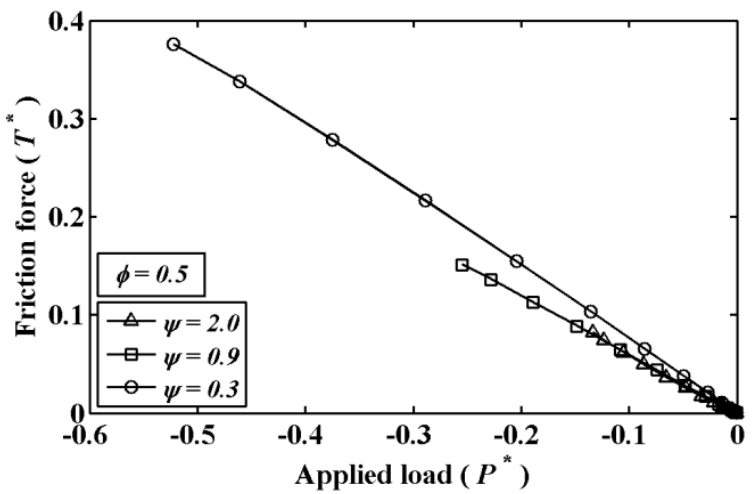

(b)

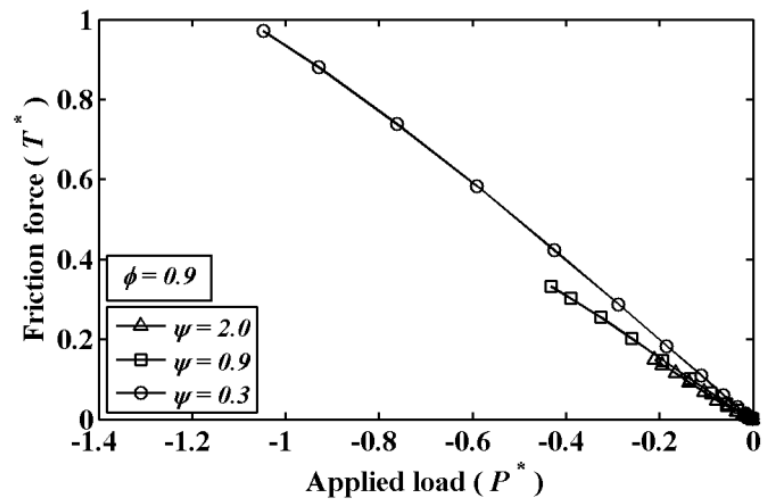

(c)

Figure 8. CEB approach formulation plotted for non-dimensional frictional force versus nondimensional applied load as a function of plasticity index $(\psi)$ in: (a) low adhesion zone $(\phi=0.1)$, (b) moderate adhesion zone $(\phi=0.5)$, (c) high adhesion zone $(\phi=0.9)$.

Now results are obtained for total applied load and total friction force on the rough surface by solving KE approach formulations. These results are plotted along with the results from CEB approach formulations in order to facilitate the comparison. In Fig. 9-11, all dotted line plots represent the CEB approach formulation results while the continuous line plots represent the KE approach formulation results.

$\mathrm{KE}$ approach of formulation makes use of mathematical expressions obtained from the finite element analysis (FEA) results while CEB approach of formulation is based upon purely analytical 
technique. Now days, it is well established fact that the FEA techniques of solving contact problems yield better results as compared to conventional analytical techniques. So in the present study it is expected that KE approach of formulation should be more accurate than CEB approach of formulation.

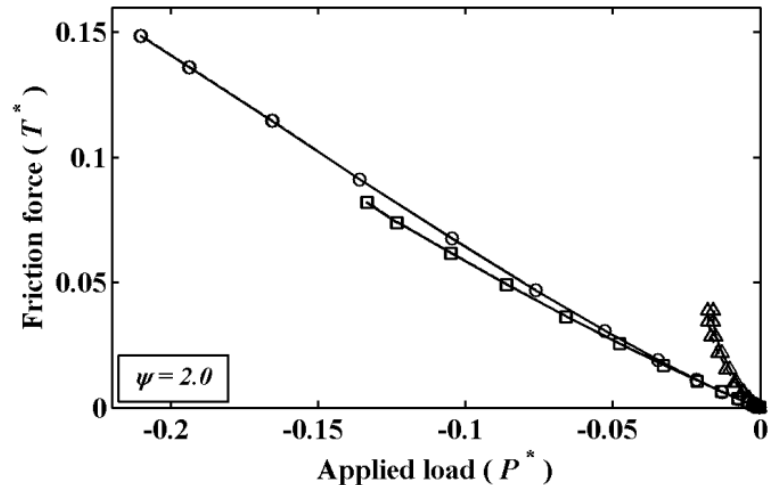

(a)

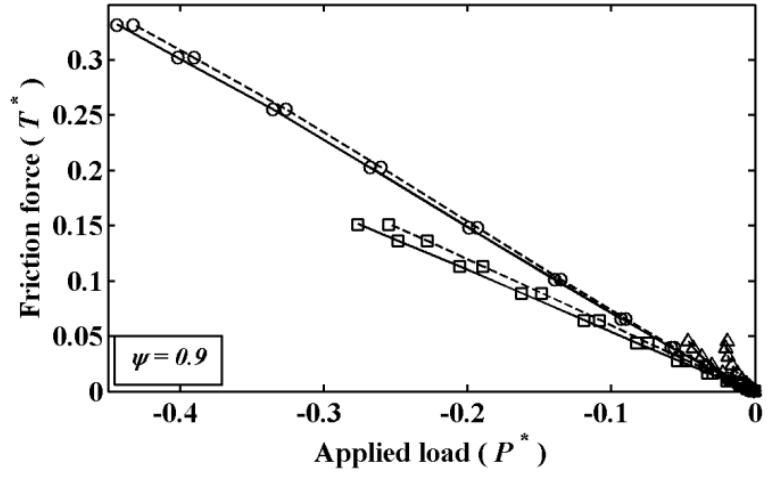

(b)

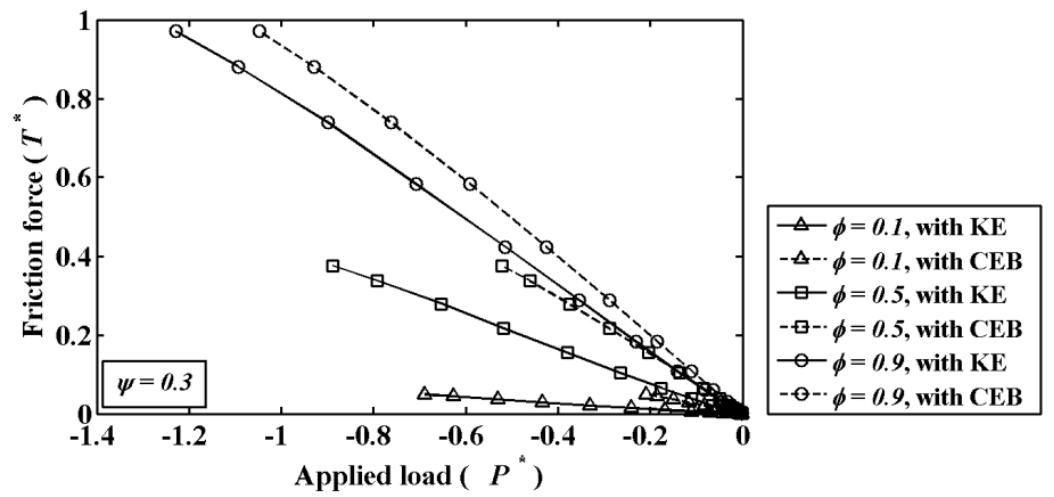

(c)

Figure 9. Comparison of results -Non-dimensional frictional force versus non-dimensional applied load as a function of adhesion index $(\phi)$ in: (a) predominantly elastic zone $(\psi=2.0)$, (b) predominantly elasto-plastic zone $(\psi=0.9)$, (c) predominantly plastic zone $(\psi=0.3)$ (Legends same for all figures).

As can be seen in all plots of Fig. 9 and Fig. 10, there exists qualitative agreement between CEB and KE approach formulation's results but quantitatively they don't match in predominantly elasto-plastic and plastic domains of deformation. This is evident from clear deviation observed between dotted and continuous line plots in all the figures except Fig. 9 (a). In Fig. 9 (b) and (c), it is observed that the results obtained by solving CEB approach formulations are overestimating the friction load for particular value of applied load while in Fig. 9 (a), the CEB approach formulation results are exactly matching with those obtained by KE approach formulation. This may be attributed to the fact that both the CEB and KE approach of formulation treat the purely elastic zone of contact in exactly same fashion and the difference exist in these two approaches for the rest of zones of deformation. 


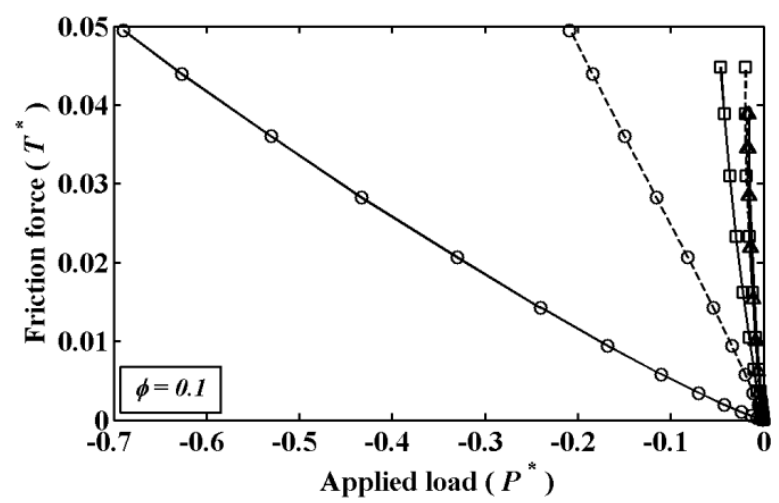

(a)

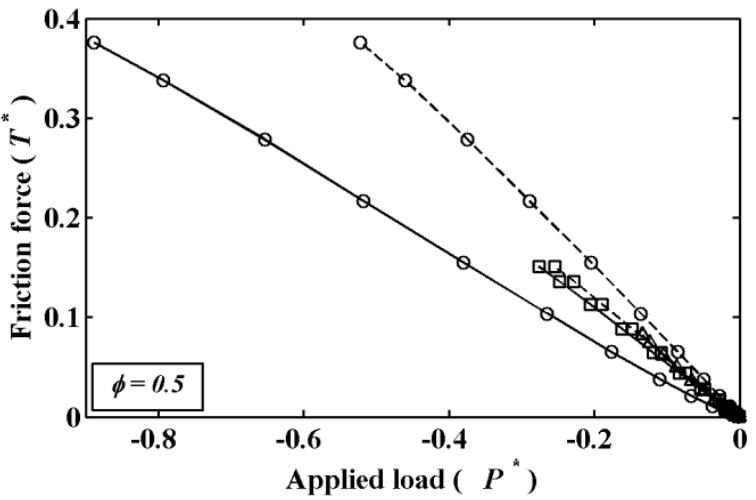

(b)

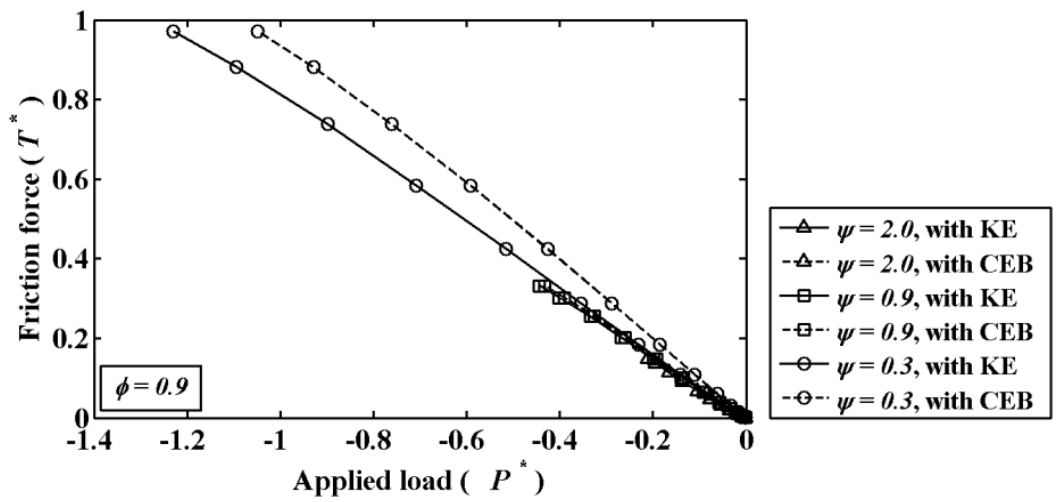

(c)

Figure 10. Comparison of results -Non-dimensional frictional force versus non-dimensional applied load as a function of plasticity index $(\psi)$ in: (a) low adhesion zone $(\phi=0.1)$, (b) moderate adhesion zone $(\phi=0.5),(\mathrm{c})$ high adhesion zone $(\phi=0.9)$.

Plots in Fig. 11 (a) and (b) show coefficient of friction versus normally applied load plots plotted in moderate $(\phi=0.5)$ and high $(\phi=0.9)$ zones of adhesion effect. From the plots it is observed that plots with $\psi=0.3$ and 0.9 have almost zero slope while that with $\psi=2.0$ have slope. Physically it means surfaces undergoing predominantly plastic type of deformation and having moderate to higher adhesion have constant coefficient of friction. Also it is observed that at a particular value of applied load, for smaller value of $\psi$, coefficient of friction values are high. Physically it means higher the tendency to deform plastically higher will be coefficient of friction. As already cleared the rough surfaces have higher tendency to undergo plastic type of deformation. Comparison of results from the two approaches shows that there is qualitative agreement between the results with the two approaches. But from quantitative agreement point of view, CEB approach formulation overestimates coefficient of friction for predominantly elasto-plastic and plastic deformations. 


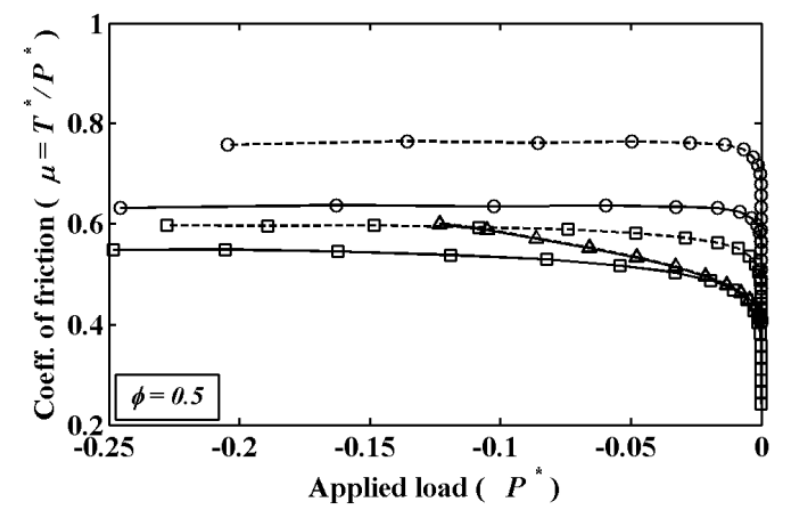

(a)

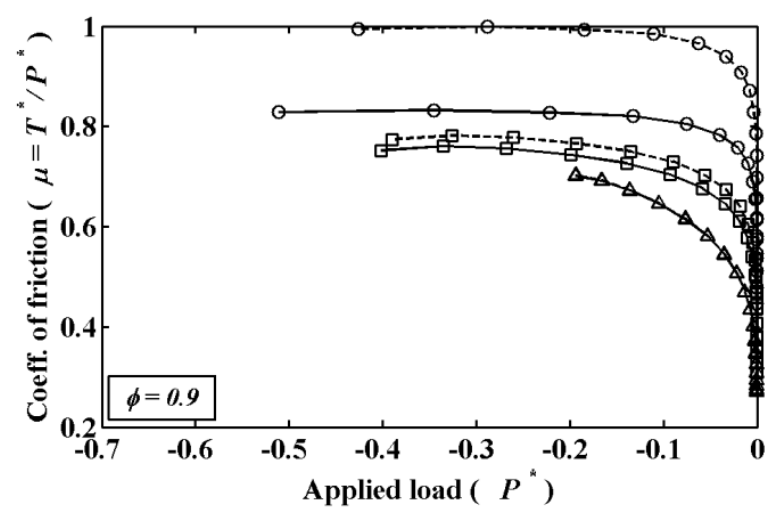

(b)

$$
\begin{aligned}
& \triangle \psi=2.0 \text {, with } \mathrm{KE} \\
& -\Delta-\psi \psi=2.0 \text {, with CEB } \\
& \text { ๑ } \psi=0.9 \text {, with } \mathrm{KE} \\
& \text {-ษ- } \psi=0.9 \text {, with CEB } \\
& \text { - } \psi=0.3 \text {, with } \mathrm{KE} \\
& -\Theta-\cdot \psi=0.3 \text {, with CEB }
\end{aligned}
$$

Figure 11. Comparison of results -Coefficient of friction versus non-dimensional applied load (Legends same for both the figures).

From the present parametric study results it is possible to locate the combinations of adhesion index and plasticity index that may yield very low coefficient of friction. Thus suitable choice of surface and material parameters for the contact of two rough surfaces can be made in order to minimize friction typically at low load and micro scale roughness situations. But there is difficulty in comparison of the results of present adhesive friction contact model which is using n-point asperities with those using conventional three point asperities. This is because such comparison demands definition of equivalence of radii of asperity from these two models. It may be noted here that the n-point asperity radius depends upon its dimension value ' $n$ ' and sampling length and changes with progression of contact. While conventional three point asperity radius definition doesn't involve sampling length and radius value is assumed to be constant throughout the progression of contact. Thus comparison of results for contact model developed with n-point asperities with those developed with conventional asperities could not be done. So the only way remaining to validate the results of models which are using n-point asperities is to conduct experimentation with the help of high end instruments like Atomic Force Microscope or Surface Force Microscope.

\section{Conclusion}

Use of n-point asperities to define surface roughness features represents more realistic picture than any other conventional method. Also incorporation of KE approach formulation for intermediate transition state of elastic-plastic deformation at the asperity level gives complete solution. Qualitative trend of the results found is same as that observed in the literature with conventional asperities. It is observed that the surfaces undergoing predominantly plastic type of deformation and having moderate to higher adhesion have constant coefficient of friction. It is possible to identify the combinations of adhesion index and plasticity index that may yield very low coefficient of friction. Thus suitable choice of surface and material parameters for the contact of two rough surfaces can be made in order to minimize friction typically at low load and micro scale roughness situations. 


\section{Notations}

$A_{0}$

$a_{(e)_{n}}$

$a_{(p)_{n}}$

$c_{n}$

$c_{(0)_{n}}$

$c_{(s)_{n}}$

$c_{(a s)_{n}}$

$c_{(a y)_{n}}$

$c_{(y)_{n}}$

E

$E_{1}, E_{2}$

$f(z)$

H

$h$

K

$K_{a d h}$

$L$

$M$

$M_{n}$

$M_{(e)_{n}}$

$M_{(e p-I)_{n}}$

$M_{(e p-I I)_{n}}$

$M_{(p)_{n}}$

$M_{(s l i p-e)_{n}}$ total nominal contact area of rough surface (sample space)

contact radius of an n-point asperity having specific ' $n$ ' dimension and in elastic mode of deformation

contact radius of an n-point asperity having specific ' $n$ ' dimension and in plastic mode of deformation

curvature coefficient corresponding to interference $\delta_{n}$ for an n-point asperity having specific ' $n$ ' dimension

apparent zero point curvature coefficient corresponding to interference $\delta_{(0)_{n}}$, for an n-point asperity having specific ' $n$ ' dimension

critical slip point curvature coefficient corresponding to interference $\delta_{(s)_{n}}$, for an npoint asperity having specific ' $n$ ' dimension

apparent critical slip point curvature coefficient corresponding to interference $\delta_{(a s)_{n}}$, for an n-point asperity having specific ' $n$ ' dimension

apparent yield point curvature coefficient corresponding to interference $\delta_{(a y)_{n}}$, for an n-point asperity having specific ' $n$ ' dimension

yield point curvature coefficient corresponding to interference $\delta_{(y)_{n}}$, for an n-point asperity having specific ' $n$ ' dimension

composite elastic modulus of an equivalent rough surface $E=\left[\left(1-v_{1}^{2}\right) / E_{1}+\left(1-v_{2}^{2}\right) / E_{2}\right]^{-1}$

elastic module of two surfaces

joint probability density functions for height ordinates

hardness of soft surface

separation between mean surface line of equivalent rough surface and rigid smooth surface

elastic constant, $K=\frac{4}{3} E$

adhesive wear coefficient

sliding distance

number of sampled points

total number of n-point asperities having specific ' $n$ ' dimension

total number of n-point asperities deformed elastically and having specific ' $n$ ' dimension

total number of n-point asperities deformed in elastic-plastic stage-I zone and having specific ' $n$ ' dimension

total number of n-point asperities deformed in elastic-plastic stage-II zone and having specific ' $n$ ' dimension

total number of n-point asperities deformed plastically and having specific ' $n$ ' dimension

total number of n-point asperities which slip in elastic zone of deformation and having specific ' $n$ ' dimension 
$M_{(s l i p-e p-I)_{n}} \quad$ total number of n-point asperities which slip in elastic-plastic stage-I zone of deformation and having specific ' $n$ ' dimension

$M_{(y i e l d-e)_{n}} \quad$ total number of n-point asperities which do not slip in elastic zone of deformation and having specific ' $n$ ' dimension

$M_{(y i e l d-e p-I)_{n}} \quad$ total number of n-point asperities which do not slip in elastic-plastic stage-I zone of deformation and having specific ' $n$ ' dimension

$n$

dimension of an n-point asperity which indicates the number of height ordinates, the asperity is comprised of

$N$

probability of existence above a defined level for an n-point asperity; all the subscripts and sub-subscripts of $N$ have the same meaning as it is associated with the subscripts and sub-subscripts of $M$

total applied load on the surface

$P_{(e)_{n}}$

$P_{(e p-I)_{n}}$

$P_{(e p-I I)_{n}}$

$P_{(p)_{n}}$

$\Delta P_{(e)_{n}}$

$\Delta P_{(e p-I)_{n}}$

$\Delta P_{(e p-I I)_{n}}$

$\Delta P_{(p)_{n}}$

$\Delta P_{(y)_{n}}$

$R_{n}$

total applied load supported by all n-point asperities having specific ' $n$ ' dimension and deformed in elastic zone

total applied load supported by all $n$-point asperities having specific ' $n$ ' dimension and deformed in elastic-plastic stage-I zone

total applied load supported by all n-point asperities having specific ' $n$ ' dimension and deformed in elastic-plastic stage-II zone

total applied load supported by all n-point asperities having specific ' $n$ ' dimension and deformed in plastic zone

applied load supported by single n-point asperity having specific ' $n$ ' dimension and deformed in elastic zone

applied load supported by single n-point asperity having specific ' $n$ ' dimension and deformed in elastic-plastic stage-I zone

applied load supported by single n-point asperity having specific ' $n$ ' dimension and deformed in elastic-plastic stage-II zone

applied load supported by single n-point asperity having specific ' $n$ ' dimension and deformed in plastic zone

critical yield point applied load for an n-point asperity having specific ' $n$ ' dimension and at critical yield point interference of $\delta_{(y)_{n}}$

$T$

radius of equivalent $n$-point asperity curve

$T_{n}$

$T_{(\text {slip-e })_{n}}$

$T_{(s l i p-e p-I)_{n}}$

$T_{(\text {yield }-e)_{n}}$

$T_{(y i e l d-e p-I)_{n}}$

total tangential load supported by surface in contact

total tangential load supported by all n-point asperities having specific ' $n$ ' dimension

tangential load supported before slip by all n-point asperities having specific ' $n$ ' dimension and deformed in elastic zone

tangential load supported before slip by all n-point asperities having specific ' $n$ ' dimension and deformed in elastic-plastic stage-I zone

tangential load supported by all $n$-point asperities which do not slip, having specific ' $n$ ' dimension and deformed in elastic zone

$\Delta T_{(s l i p-e)_{n}} \quad$ tangential load supported before slip by single n-point asperity having specific ' $n$ ' dimension and deformed in elastic zone

$\Delta T_{(s l i p-e p-I)_{n}} \quad$ tangential load supported before slip by single n-point asperity having specific ' $n$ ' dimension and deformed in elastic-plastic stage-I zone 
$\Delta T_{(y i e l d-e)_{n}} \quad$ tangential load supported by single n-point asperity which do not slip, having specific ' $n$ ' dimension and deformed in elastic zone

$\Delta T_{(y i e l d-e p-I)_{n}}$ tangential load supported by single n-point asperity which do not slip, having specific ' $n$ ' dimension and deformed in elastic plastic stage-I zone

$\Delta x$ sampling length, $\Delta x=\beta^{*}(-\operatorname{In} \rho)$

$z_{n}$ height of $\mathrm{n}^{\text {th }}$ ordinate from mean surface line

$z_{n}^{*}$

$=\frac{z_{n}}{\sigma}$

$z_{0}$

height of equivalent n-point asperity curve

$z_{0}^{*}$

$=\frac{z_{0}}{\sigma}$

$\beta$

correlation length

$\gamma$

work of adhesion

$\delta_{n}$

$\delta_{(0)_{n}}$ interference of an n-point asperity having specific ' $n$ ' dimension

$\delta_{(y)_{n}}$ apparent zero interference of an n-point asperity having specific ' $n$ ' dimension yield point interference of an n-point asperity having specific ' $n$ ' dimension

$\delta_{(y a)_{n}}$

$\delta_{(s)_{n}}$

$\delta_{(s a)_{n}}$ apparent yield point interference of an n-point asperity having specific ' $n$ ' dimension slip point interference of an n-point asperity having specific ' $n$ ' dimension

$\delta_{n}^{*}$ apparent slip point interference of an n-point asperity having specific ' $n$ ' dimension $=\frac{\delta_{n}}{\sigma}$; non dimensional interference of an n-point having specific ' $n$ ' dimension

$\phi$ $=\frac{\gamma \beta}{E \sigma^{2}} ;$ adhesion index

$\psi$

$=\frac{H \beta}{E \sigma} ;$ plasticity index

$\rho$ correlation coefficient

$\sigma$ root mean square roughness or standard deviation of height ordinates Poisson's ratio $=(n-2) z_{0}^{*}-(n-4) c / 6$

asterisk (*) mark as All values of different parameters with asterisk as their superscript indicate superscript normalized (non-dimensional) values. 


\section{References}

[1] R.D. Mindlin, Compliance of elastic bodies in contact, J. Appl. Mech. 16 (1949) 259-268.

[2] F.P. Bowden, D. Tabor, The friction and lubrication of solids, vol. 2, Oxford Univ Press, Oxford, 1964.

[3] D. Tabor, Friction - The present state of our understanding, J. Lub. Tech. 103(2) (1981) 169179.

[4] W.R. Chang, I. Etsion, D.B. Bogy, Adhesion model for metallic rough surfaces, ASME J. Trib. 110 (1988) 50-56.

[5] G.M. Hamilton, Explicit equations for the stresses beneath a sliding spherical contact, Proc. Inst. Mech. Eng. 197C (1983) 53-59.

[6] L. Kogut, I. Etsion, A finite element based elastic-plastic model for the contact of rough surfaces, Trib. Trans. 46(3) (2003) 383-390.

[7] S.K.R. Chowdhury, P. Ghosh, Adhesion and adhesional friction at the contact between solids, Wear. 174 (1994) 9-19.

[8] A.R. Savkoor, G.A.D. Briggs, The effect of tangential force on the contact of elastic solids in adhesion, Proc. R. Soc. Lond. A. 356 (1977) 103-114.

[9] W.R. Chang, I. Etsion, D.B. Bogy, An elastic-plastic model for the contact of rough surfaces, ASME J. Trib. 109 (1987) 257-263.

[10] D.G. Evseev, B.M. Medvedev, G.G. Grigoriyan, Modification of the elastic-plastic model for the contact of rough surfaces, Wear. 150 (1991) 79-88.

[11] Y. Zhao, D.M. Maietta, L. Chang, An asperity microcontact model incorporating the transition from elastic deformation to fully plastic flow, ASME J. Trib. 122(1) (2000) 86-93.

[12] S. Kucharski et al., Finite elements model for the contact of rough surfaces, Wear. 177 (1994) $1-13$.

[13] L. Kogut, I. Etsion, Elastic-plastic contact analysis of a sphere and a rigid flat, J. Appl. Mech. 69(5) (2002) 657-662.

[14] D.M. Schaefer et al., Surface roughness and its influence on particle adhesion using atomic force techniques, J. Adhesion Sc. Tech. 9(8) (1995) 1049-1062.

[15] K.L. Johnson, Adhesion and friction between a smooth elastic spherical asperity and a plane surface, Proc. R. Soc. Lond. A. 453 (1956) 163-179.

[16] Y. I. Rabinovich et al., Adhesion between nanoscale rough surfaces: I. Role of asperity geometry, J. Coll. Interface Sc. 232(1) (2000) 10-16.

[17] A. Hariri, J.W. Zu, R. Ben Mrad, n-Point asperity model for contact between nominally flat surfaces, ASME J. Trib. 128 (2006) 505-514.

[18] A. Hariri, J.W. Zu, R. Ben Mrad, Modeling of elastic/plastic contact between nominally flat rough surfaces using an n-point asperity model, ASME J. Trib. 128 (2006) 876-885.

[19] A.K. Waghmare, P. Sahoo, A study of elastic-plastic contact of rough surfaces using n-point asperity model, Procedia Mat. Sc. 5 (2014) 1716-1725.

[20] A.K. Waghmare, P. Sahoo, Elastic-plastic adhesive contact of rough surfaces based on accurate FEA study using n-point asperity model, Int. J. Surface Eng. and Interdisciplinary Mat. Sc. 2(2) (2014) 1-22. 
[21] A. K. Waghmare, P. Sahoo, Adhesive friction at the contact between rough surfaces using npoint asperity model, Engineering Science and Technology, an International Journal. 18(3) (2015) 463-474.

[22] A.K. Waghmare, P. Sahoo, Friction analysis at elastic-plastic contact of rough surfaces using n-point asperity model, Proc. Inst. Mech. Eng. Part J: J. Eng. Trib. 230(10) (2016) 12581272.

[23] P. Sahoo, A.K. Waghmare, Adhesive friction based on finite element study and n-point asperity model, J. Phys.: Conf. Series. 738 (1) (2016) 012116.

[24] J.A. Greenwood, J.B.P. Williamson, Contact of nominally flat surfaces, Proc. R. Soc. Lond. A. 295 (1966) 300-319.

[25] J.F. Archard, Elastic deformation and the laws of friction, Proc. R. Soc. Lond. A. 243 (1957) 190-205.

[26] J.A. Greenwood, J.J. Wu, Surface roughness and contact: an apology, Meccanica. 36(6) (2001) 617-630.

[27] K.L. Johnson, K. Kendall, A.D. Roberts, Surface energy and the contact of elastic solids, Proc. R. Soc. Lond. A. 324 (1971) 301-313.

[28] S.K.R. Chowdhury, H.M. Pollock, Adhesion between metal surfaces: The effect of surface roughness, Wear. 66 (1981) 307-321.

[29] D. Tabor, The Hardness of Metals, Oxford University Press, Oxford, 1951.

[30] P. Sahoo, A. Mitra, K. Saha, Elastic-plastic adhesive contact of rough surfaces using n-point asperity model, J. Physics D: Appl. Phys. 42 (2009) 1-13.

[31] K.L. Johnson, Adhesion at the contact of solids, in W.T. Koiter (Ed.), 4th IUTAM Congress Proceedings on Theoretical and Applied Mechanics, Amsterdam, North-Holland, 1976, pp. $133-143$.

[32] L. Kogut, I. Etsion, A static friction model for elastic-plastic contacting rough surfaces, ASME J. Trib. 126(1) (2004) 34-40.

[33] J.A. Greenwood, J.H. Tripp, The contact of two nominally flat rough surfaces, Proc. Inst. Mech. Eng. 185 (1971) 625-633.

[34] M. O'Callaghan, M.A. Cameron, Static contact under load between nominally flat surfaces in which deformation is purely elastic, Wear. 36 (1976) 79-97.

[35] J.N. Israelachvili, Intermolecular and surface forces, Academic press, 2011.

[36] D.J. Whitehouse, J.F. Archard, The properties of random surfaces of significance in their contact, Proc. R. Soc. Lond. A. 316 (1970) 97-121. 